\title{
ON UNIVERSAL RELATIONS IN GAUGE THEORY
}

\author{
SELMAN AKBULUT
}

\begin{abstract}
In this paper we study the algebraic topology of gauge group, and as a corollary we deduce some universal relations among Donaldson polynomials of smooth 4-manifolds.
\end{abstract}

\section{INTRODUCTION}

Let $P \longrightarrow X^{4}$ be a principal $S U(2)$ or $S O(3)$ bundle over a smooth simply connected 4-manifold $X$. Hence in case $P$ is an $S O(3)$-bundle, it lifts to a $U(2)$ bundle. It follows that when $w_{2}(X)=w_{2}(P)$ the parity of $c_{2}(P)$ is well defined number (i.e. it is independent of the lifting).

Recall that (e.g. [DK]) the space $\mathcal{B}^{*}(P)$ of gauge equivalence classes of irreducible connections in $P$ is an infinite dimensional manifold with nontrivial topology. $\mathcal{B}^{*}(P)$ also admits a natural $S O(3)$-bundle $\bar{\xi} \rightarrow \mathcal{B}^{*}(P)$ which is usually called the base point fibration. For a generic choice of a metric $g$ on $X$, the gauge equivalence classes of anti-self-dual connections $\mathcal{M}(P, g) \subset \mathcal{B}^{*}(P)$ forms a smooth orientable (possibly noncompact) finite dimensional submanifold, and its bordism class in $\mathcal{B}^{*}(P)$ is independent of the metric $g$.

When the dimension $b_{2}^{+}(X)$ of the positive definite part of $H_{2}(X)$ is odd then the dimension of $\mathcal{M}(P, g)$ is an even integer $2 d=2 d_{k}=8 k-3\left(1+b_{2}^{+}(X)\right)$, where $k=-p_{1}(P) / 4$. For $a_{1}, \ldots, a_{d} \in H_{2}(X)$ Donaldson defines invariants $\Phi_{d}\left(a_{1}, \ldots, a_{d}\right)$ by associating to each $a_{i}$ a codimension two submanifold $V_{i} \subset \mathcal{M}(P, g)$ and counting the "intersection number"

$$
\mathcal{M}(P, g) \cap V_{1} \cap \cdots \cap V_{d} .
$$

These invariants are usually assembled into a single homogeneous polynomial: $\Phi_{d}=$ $\Phi_{X, d}=\sum \Phi_{k}\left(a_{1}, \ldots, a_{d}\right) a_{1}^{*} \cdots a_{d}^{*}$ in the dual homology classes. There is yet another invariant $\Phi_{d}\left(a_{1}, \ldots, a_{d-2}, 1\right)$ which is defined by the evaluation:

$$
\left\langle p_{1}(\bar{\xi}), \mathcal{M}(P, g) \cap V_{1} \cap \cdots \cap V_{d-2}\right\rangle .
$$

In this paper we compute some higher homotopy groups of $\mathcal{B}^{*}(P)$ generalizing the fundamental group calculation of [AMR]. We study the topology of $\mathcal{B}^{*}(P)$ and discuss its Postnikov tower. By relating the $k$ invariants of the Postnikov tower of $\mathcal{B}^{*}(P)$ to the homotopy groups coming from the "ends" of $\mathcal{M}(P, g)$, we show that the various Donaldson polynomial invariants have to satisfy some universal relations. These calculations are carried by evaluating certain secondary cohomology operations associated to the Postnikov tower of the classifying space $B U(2)$,

Received by the editors November 1, 1994

1991 Mathematics Subject Classification. Primary 57R19; Secondary 58D27.

Partially supported by the N.S.F. 
by the methods of Emery Thomas [T]. The use of $k$-invariants of $\mathcal{B}^{*}(P)$ brings a special importance to its homotopy groups, by enabling us to relate them to the polynomials $\Phi_{d}$. The $k$-invariants are calculated by proving some divisibility conditions for certain "universal" cohomology classes of $\mathcal{B}^{*}(P)$. Our motivation for this divisibility theorem mainly comes from Masbaum's resuts in $[\mathrm{M}]$.

The question of whether these polynomial invariants satisfy some universal relations was raised by Donaldson. Friedman and Morgan showed that in the case of complex surfaces with "large diffeomorphism group" the Donaldson invariants are polynomials in their first Chern class and the intersection form. In $[R]$ Ruan showed that, under some conditions on $P$, a certain combination of the invariants $\Phi_{d}$ have to be even. Later this was generalized as

Theorem $([\mathrm{AMR}])$. If $c_{2}(P)$ is odd and $w_{2}(P)=w_{2}(X)$, then for all classes $\alpha_{i} \in H^{2}(X)$ with $\alpha_{i}^{2} \equiv 0(\bmod 2)$ the Donaldson invariants satisfy:

$$
\sum_{i<j} q\left(a_{i}, a_{j}\right) \Phi_{d}\left(a_{1}, \ldots, \widehat{a}_{i}, \ldots, \widehat{a}_{j}, \ldots, a_{d+2}\right)=0 \quad(\bmod 2) .
$$

where $q\left(a_{i}, a_{j}\right)$ denotes the intersection number of $a_{i}$ with $a_{j}$.

In [FS] Fintushel and Stern proved some mod 2 vanishing results for the invariants $\Phi_{d}$. Our main result gives a way of relating different degree polynomial invariants $\Phi_{d}$ and $\Phi_{d+4}$ to each other:

Theorem 5.4. If $P \rightarrow X$ is an SO(3)-bundle over a closed smooth simply connected 4-manifold, assume that $c_{2}(P)$ is even when $w_{2}(P)=w_{2}(X)$. Then for any basis $b_{1}, \ldots, b_{n}$ of $H_{2}(X)$ and $a_{1}, \ldots, a_{d+2} \in H_{2}(X)$, we have

$$
\begin{aligned}
& \sum_{i, j} \bar{q}\left(b_{i}, b_{j}\right) \Phi_{d+4}\left(a_{1}, \ldots, a_{d+2}, b_{i}, b_{j}\right)+(d+1) \Phi_{d+4}\left(a_{1}, \ldots, a_{d+2}, 1\right) \\
& \equiv \pm d(d-1) \sum_{i<j} q\left(a_{i}, a_{j}\right) \Phi_{d}\left(a_{1}, \ldots, \widehat{a}_{i}, \ldots, \widehat{a}_{j}, \ldots, a_{d+2}\right) \quad(\bmod 3)
\end{aligned}
$$

where $\left(\bar{q}\left(b_{i}, b_{j}\right)\right)$ denotes the inverse of the intersection matrix $\left(q\left(b_{i}, b_{j}\right)\right)$.

The sign ambiguity in the above identity can be decided by evaluating it on an example, which we did not do in this paper. We also prove some $\bmod p$ congruences between Donaldson polynomials. Some of the calculations of this paper are generalized in $[\mathrm{A}]$. We hope to explore further generalizations in a future paper. If one views Donaldson invariants as a kind of nonlinear cohomology, then these relations may be thought of as "cohomology operations" of this theory. At the end we apply these results to the complex surfaces with large diffeomorphism group to get more concrete conclusions. We would like to thank Y. Ruan for his many motivating questions and enthusiastic support of this research. Finally I would like to thank John Havlicek for reading this paper and making valuable suggestions.

\section{Topology of $\mathcal{B}^{*}(P)$}

Let $X$ be a closed smooth oriented 4-manifold with $\pi_{1}(X)=0$, and $P \longrightarrow X$ be a principal $G$-bundle, where $G=S O(3)$, or $S U(2)$. Let $C(G)$ denote the center of $G$. Let $\mathcal{A}(P)$ be the space of connections on $P$, and let $\mathcal{A}^{*}(P)$ denote the subspace of the irreducible connections on $P$. Let $\mathcal{G}(P)$ be the gauge group acting on $\mathcal{A}(P)$. 
The quotient $\hat{\mathcal{G}}(P)=\mathcal{G}(P) / C(G)$ acts on $\mathcal{A}^{*}(P)$ freely giving us the principal fibration:

$$
\hat{\mathcal{G}}(P) \longrightarrow \mathcal{A}^{*}(P) \longrightarrow \mathcal{B}^{*}(P)
$$

where $\mathcal{B}^{*}(P)=\mathcal{A}^{*}(P) / \hat{\mathcal{G}}(P)$. For brevity the dependence of these spaces on $P$ sometimes will be dropped from their notation. Since the total space of the fibration is contractible, we can identify $\mathcal{B}^{*}(P)=B_{\hat{\mathcal{G}}}$. Recall that the following proposition allows us to compute the homotopy groups of $\mathcal{B}^{*}(P)$ (cf. [AMR]).

Proposition 2.1. There is a weak homotopy equivalence:

$$
\mathcal{B}^{*}(P) \simeq \operatorname{Map}^{P}(X, B S O(3))
$$

where the right-hand side denotes the component of the space of (unbased) maps inducing the bundle P.

The evaluation map ev : $X \times \operatorname{Map}^{P}(X, B S O(3)) \longrightarrow B S O(3)$ induces an $S O(3)$ bundle $\xi \rightarrow X \times \mathcal{B}^{*}$. Now let $\mu: H_{2}(X) \rightarrow H^{2}\left(\mathcal{B}^{*}\right)$ be the usual $\mu$-map, defined by the homology slant product $\mu(a)=-p_{1}(\xi) / a$. To be precise Donaldson's $\mu$ map which we will call $\mu^{*}$ is defined to be $\mu / 4$. In order to make $\mu^{*}$ an integral class we have to define it on a smaller subgroup:

$$
\mu^{*}: A_{2}(X)=\left\{a \in H_{2}(X) \mid\left\langle w_{2}(P), a\right\rangle=0\right\} \rightarrow H^{*}\left(\mathcal{B}^{*} ; \mathbf{Z}\right) .
$$

$2 \mu^{*}$ is integral on $H_{2}(X)$. It is by this map $\mu^{*}$ which the Donaldson's invariants are defined (e.g. $[\mathrm{DK}])$.

For a basis $a_{1}, a_{2}, \ldots, a_{n}$ of $H_{2}(X)$ we define

$$
\Omega=\frac{1}{4} \sum_{i, j} \bar{q}\left(a_{i}, a_{j}\right) \mu\left(a_{i}\right) \mu\left(a_{j}\right) \in H^{4}\left(\mathcal{B}^{*} ; \mathbf{Z}\right) .
$$

Here $\left(\bar{q}\left(a_{i}, a_{j}\right)\right)$ denotes the inverse of the intersection matrix $\left(q\left(a_{i}, a_{j}\right)\right)$. It is easy to check that $\Omega$ is independent of the chosen basis, and is a natural class in the sense of $[\mathrm{M}]$. This means that if we write $\Omega=\Omega(X)$ then for any degree one map $f: X^{\prime} \rightarrow X$ the induced map $F: \operatorname{Map}^{P}(X, B S O(3)) \rightarrow \operatorname{Map}^{P^{\prime}}\left(X^{\prime}, B S O(3)\right)$, where $P^{\prime}=f^{*}(P)$, has the property: $F^{*}\left(\Omega\left(X^{\prime}\right)\right)=\Omega(X)$.

By restriction, the bundle $\xi$ gives a bundle $\bar{\xi} \longrightarrow \mathcal{B}^{*}$ which is usually called the base point fibration. Let $\bar{p}_{1} \in H^{4}\left(\mathcal{B}^{*} ; \mathbf{Z}\right)$ denote the first Pontryagin class of $\bar{\xi}$, i.e. $\bar{p}_{1}=-\mu(1)$. Let $p_{1}$ denote the first Pontryagin number of $P$, i.e $p_{1}=\left\langle p_{1}(P),[X]\right\rangle$ where $[X]$ is the generator of $H_{4}(X)$. The following theorem is essentially a certain generalization of a result of Masbaum $[\mathrm{M}]$.

Proposition 2.2. If $X$ is even then $\Omega-p_{1} \bar{p}_{1} \in H^{4}\left(\mathcal{B}^{*} ; \mathbf{Z}\right)$ is divisible by 6 . Furthermore, if $\pi_{1}\left(\mathcal{B}^{*}\right)=0$ then $\Omega-p_{1} \bar{p}_{1}$ is divisible by 12 .

Proof. Let $\bar{P} \longrightarrow X$ be a lifting of $P$ to a $U(2)$-bundle. Principal fibrations:

$$
S^{1} \rightarrow U(2) \rightarrow S O(3) \rightarrow B S^{1} \rightarrow B U(2) \longrightarrow B S O(3)
$$

induce a principal fibration (action is the pointwise multiplication of maps):

$$
\operatorname{Map}^{0}(X, K(\mathbf{Z}, 2)) \rightarrow \operatorname{Map}^{\bar{P}}(X, B U(2)) \stackrel{\pi}{\longrightarrow} \operatorname{Map}^{P}(X, B S O(3))
$$

where $\operatorname{Map}^{0}(X, K(\mathbf{Z}, 2))$ means the component of the maps inducing 0 in the second cohomology (since $B S^{1}=K(\mathbf{Z}, 2)$ ). The fiber of this fibration is $K(\mathbf{Z}, 2)$. So, we get a fibration $K(\mathbf{Z}, 2) \rightarrow \tilde{\mathcal{B}} \stackrel{\pi}{\longrightarrow} \mathcal{B}^{*}$, where $\tilde{\mathcal{B}}=\operatorname{Map}^{\bar{P}}(X, B U(2))$. 
As above we can consider the $U(2)$-bundle $\eta \rightarrow X \times \tilde{\mathcal{B}}$ induced by the evaluation map $X \times \tilde{\mathcal{B}} \rightarrow B U(2)$, and define the corresponding map $\tilde{\mu}: H_{2}(X) \rightarrow H^{2}(\tilde{\mathcal{B}})$, by taking the slant product with $-p_{1}(\eta)$. Let

$$
\tilde{\Omega}=\frac{1}{4} \sum_{i, j} \bar{q}\left(a_{i}, a_{j}\right) \tilde{\mu}\left(a_{i}\right) \tilde{\mu}\left(a_{j}\right) \in H^{4}(\tilde{\mathcal{B}} ; \mathbf{Z}) .
$$

Similarly by restricting $\eta$ we get a base point fibration $\bar{\eta} \longrightarrow \tilde{\mathcal{B}}$. Since $p_{1}(\eta)=$ $p_{1}\left(\pi^{*} \xi\right)$, we have $\pi^{*}\left(\Omega-p_{1} p_{1}(\bar{\xi})\right)=\tilde{\Omega}-p_{1} p_{1}(\bar{\eta})$. We claim $\pi^{*}: H^{4}\left(\mathcal{B}^{*}\right) \rightarrow H^{4}(\tilde{\mathcal{B}})$ is split injective when $\pi_{1}\left(\mathcal{B}^{*}\right)=0$, and may be multiplication by 2 in general. By this claim, Proposition 2.2 follows if we show $\tilde{\Omega}-p_{1} p_{1}(\bar{\eta})$ is divisible by 12 .

Let us first prove the claim: First of all the fibration $K(\mathbf{Z}, 2) \rightarrow \tilde{\mathcal{B}} \stackrel{\pi}{\longrightarrow} \mathcal{B}^{*}$ is induced from $K(\mathbf{Z}, 2) \rightarrow B U(2) \longrightarrow B S O(3)$ by the evaluation map, it has a constant coefficient system. By the spectral sequence of the fibration $K(\mathbf{Z}, 2) \rightarrow$ $\tilde{\mathcal{B}} \stackrel{\pi}{\longrightarrow} \mathcal{B}^{*}, \pi^{*}$ is given by the following composition:

$$
\pi^{*}: H^{4}\left(\mathcal{B}^{*}\right)=E_{2}^{4,0}=E_{\infty}^{4,0}=F^{4,0}=F^{3,1} \subset F^{2,2}=F^{1,3} \subset F^{0,4}=H^{4}(\tilde{\mathcal{B}})
$$

where $E_{\infty}^{p, q}=F^{p, q} / F^{p+1, q-1}$. The following is the $E_{2}$ of the spectral sequence:

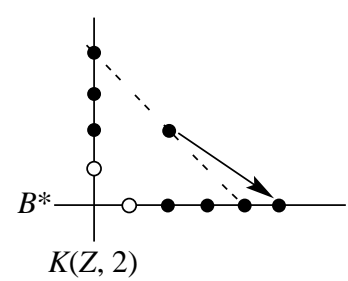

FiguRE 1

By Proposition $4.1 \pi_{1}\left(\mathcal{B}^{*}\right)=0$ or $\mathbf{Z}_{2}, E_{\infty}^{0,4}$ is free and $E_{\infty}^{2,2}$ is free up to a $\mathbf{Z}_{2}$ summand. Then, by the exactness of $0 \rightarrow E_{\infty}^{2,2} \rightarrow H^{4}(\tilde{\mathcal{B}}) / H^{4}\left(\mathcal{B}^{*}\right) \rightarrow E_{\infty}^{0,4} \rightarrow 0$ the group $H^{4}(\tilde{\mathcal{B}}) / H^{4}\left(\mathcal{B}^{*}\right)$ is free up to a $\mathbf{Z}_{2}$ summand or free when $\pi_{1}\left(\mathcal{B}^{*}\right)=\mathbf{Z}_{2}$. Hence the proof of the claim follows from the exact sequence

$$
0 \rightarrow H^{4}\left(\mathcal{B}^{*}\right) \stackrel{\pi^{*}}{\longrightarrow} H^{4}(\tilde{\mathcal{B}}) \rightarrow H^{4}(\tilde{\mathcal{B}}) / H^{4}\left(\mathcal{B}^{*}\right) \rightarrow 0
$$

Now we have to show that $\tilde{\Omega}-p_{1} \bar{p}_{1}$ is divisible by 12 . A similar fact was proved in $[\mathrm{M}]$ in the case of $B S U(2)$. We will modify [M] to fit our $B U(2)$ case.

By naturality, it suffices to prove this for $X=\sharp_{g} S^{2} \times S^{2}[\mathrm{M}]$. Consider the commutative diagram obtained by stabilizing the evaluation maps:

$$
\begin{array}{cll}
X \times \operatorname{Map}^{\bar{P}}(X, B U(2)) & \stackrel{\eta}{\longrightarrow} & B U(2) \\
\downarrow 1 \times j & & \downarrow j \\
X \times \operatorname{Map}^{\bar{P}}(X, B U) & \stackrel{\tilde{\eta}}{\longrightarrow} & B U
\end{array}
$$

Since $B U \simeq B S U \times K(\mathbf{Z}, 2)$, by calling $k=c_{2}(\bar{P})$ we get

$$
\begin{aligned}
\operatorname{Map}^{\bar{P}}(X, B U) & \simeq \operatorname{Map}^{k}(X, B S U) \times \operatorname{Map}(X, K(\mathbf{Z}, 2)) \\
& \simeq \operatorname{Map}^{k}(X, B S U) \times K(\mathbf{Z}, 2)
\end{aligned}
$$

By $[\mathrm{M}]$ there is a homotopy equivalence:

$$
\phi: \operatorname{Map}^{k}(X, B S U) \stackrel{\simeq}{\longrightarrow} B S U \times(B U)^{2 g} \times B U
$$


given by $\phi(f)=(f(p t), P(f), Q(f))$ for some $P$ and $Q$. The evaluation map $\eta_{0}: X \times \operatorname{Map}^{k}(X, B S U) \rightarrow B S U$ corresponds to

$$
\left(x, z,\left(y_{1}, y_{1}^{\prime}, \ldots, y_{g}, y_{g}^{\prime}\right), y\right) \longrightarrow z \cdot \prod_{i=1}^{g} F\left(r_{i}(x), y_{i}\right) \cdot F\left(r_{i}^{\prime}(x), y_{i}^{\prime}\right) \cdot \tilde{F}([x], y)
$$

where $F: S^{2} \times B U \rightarrow B S U, \tilde{F}: S^{4} \times B U \rightarrow B S U$ are adjoints of the Bott equivalences $B U \simeq \Omega^{2} B S U, B U \simeq \Omega_{k}^{4} B S U$, and $r_{i}, r_{i}^{\prime}: X \rightarrow S^{2}$ are the maps obtained by first collapsing $X=\sharp_{g} S^{2} \times S^{2}$ to the $i$ th factor $S^{2} \times S^{2}$ and then projecting to the first and the second $S^{2}$ factors respectively. Also $[x]$ denotes the image of $x \in X$ in $X / \bigvee_{i=1}^{g} S^{2} \vee S^{2} \approx S^{4}$.

If $c$ denotes the total Chern class and $s_{n}$ denotes the Newton polynomials, and $\sigma_{i} \in H^{i}\left(S^{i} ; \mathbf{Z}\right), i=2,4$, are the standard generators then a routine calculation (cf. $[\mathrm{M}])$ gives

$$
\begin{aligned}
& F^{*}(c)=1+\sigma_{2} \times A, \text { with } A=\sum_{n \geq 1}(-1)^{n+1} s_{n}\left(c_{1}, c_{2}, \ldots\right), \\
& \tilde{F}^{*}(c)=1+\sigma_{4} \times B, \text { with } B=k+\sum_{n \geq 1}(-1)^{n+1}(n+1) s_{n}\left(c_{1}, c_{2}, \ldots\right),
\end{aligned}
$$

Notice in the splitting $B U \simeq B S U \times K(\mathbf{Z}, 2)$ the universal bundle $\omega$ corresponds to $\omega_{0} \oplus L$, where $\omega_{0}=\omega \oplus L^{-1}$, and $L=\operatorname{det}(\omega)$. Hence the bundle induced by $\tilde{\eta}$ splits as: $\tilde{\eta}=\eta_{0} \oplus L$. So the total Chern class is $c(\tilde{\eta})=c(L) c\left(\eta_{0}\right)$.

Let $\left\{a_{1}, a_{1}^{\prime}, \ldots, a_{g}, a_{g}^{\prime}\right\}$ be the standard basis of $H^{2}(X)$, and for the sake of simplicity let $[X]$ also denote the generator of $H^{4}(X)$. Since the multiplication on $B S U$ is induced by the Whitney sum of bundles the total Chern class of $\tilde{\eta}$ can be written as

$$
\begin{aligned}
c(\tilde{\eta}) & =c(L)(1 \times c) \prod_{i=1}^{g}\left(1+a_{i} \times A_{i}\right)\left(1+a_{i}^{\prime} \times A_{i}^{\prime}\right)(1+[X] \times B) \\
& =c(L)\left[1 \times c+\sum a_{i} \times c A_{i}+\sum a_{i}^{\prime} \times c A_{i}^{\prime}+[X] \times c\left(B+\sum A_{i} A_{i}^{\prime}\right)\right] .
\end{aligned}
$$

Here classes $c, A_{i}, A_{i}^{\prime}$, and $B$ in $H^{*}\left(\operatorname{Map}^{k}(X, B S U)\right)$ correspond in the obvious way to the components of $\operatorname{Map}^{k}(X, B S U) \simeq B S U \times(B U)^{2 g} \times B U$. Here we also used $a_{i} a_{i}^{\prime}=\delta_{i j}[X]$ and $a_{i} a_{j}=0=a_{i}^{\prime} a_{j}^{\prime}$.

Now write: $c(L)=\alpha \times 1+1 \times \tilde{\beta}$, where $\alpha=\sum \lambda_{i} a_{i}+\sum \lambda_{i}^{\prime} a_{i}^{\prime}$ and $\tilde{\beta}=1+\beta$. By calling $\tilde{c}=\tilde{\beta} c$ we get

$$
\begin{aligned}
c(\tilde{\eta})= & 1 \times \tilde{c}+\sum a_{i} \times \tilde{c} A_{i}+\sum a_{i}^{\prime} \times \tilde{c} A_{i}^{\prime}+[X] \times \tilde{c}\left(B+\sum A_{i} A_{i}^{\prime}\right) \\
& +\sum[X] \times c \lambda_{i}^{\prime} A_{i}+\sum[X] \times c \lambda_{i} A_{i}^{\prime}+\alpha \times c \\
= & 1 \times \tilde{c}+\sum a_{i} \times c\left(\tilde{\beta} A_{i}+\lambda_{i}\right)+\sum a_{i}^{\prime} \times c\left(\tilde{\beta} A_{i}^{\prime}+\lambda_{i}^{\prime}\right) \\
& +[X] \times c\left(\tilde{\beta} B+\tilde{\beta} \sum A_{i} A_{i}^{\prime}+\sum \lambda_{i}^{\prime} A_{i}+\sum \lambda_{i} A_{i}^{\prime}\right) .
\end{aligned}
$$

Now express the total Chern class of the bundle $\eta \longrightarrow X \times \operatorname{Map}^{\bar{P}}(X, B U(2))$

$$
c(\eta)=1 \times \bar{c}+\sum a_{i} \times b_{i}+\sum a_{i}^{\prime} \times b_{i}^{\prime}+c(\bar{P}) \times 1
$$


where $c(\bar{P})=c_{1}(\bar{P})+c_{2}(\bar{P})$. By expressing in the basis $c_{1}(\bar{P})=\sum \mu_{i} a_{i}+\sum \mu_{i}^{\prime} a_{i}^{\prime}$ and $c_{2}(\bar{P})=k[X]$ we can write

$$
c(\eta)=1 \times \bar{c}+\sum a_{i} \times\left(b_{i}+\mu_{i}\right)+\sum a_{i}^{\prime} \times\left(b_{i}^{\prime}+\mu_{i}^{\prime}\right)+k[X] \times 1 .
$$

From commutativity of the diagram $\left(^{*}\right)$ we have $J^{*}(c(\tilde{\eta}))=c(\eta)$, where $J=1 \times j$. Hence $j^{*}(\tilde{c})=j^{*}(\tilde{\beta} c)=\bar{c}$ and

$$
\begin{array}{lll}
j^{*}\left(c\left(\tilde{\beta} A_{i}+\lambda_{i}\right)\right) & = & b_{i}+\mu_{i}, \\
j^{*}\left(c\left(\tilde{\beta} A_{i}^{\prime}+\lambda_{i}^{\prime}\right)\right) & = & b_{i}^{\prime}+\mu_{i}^{\prime}, \\
j^{*}\left(c\left(\tilde{\beta} B+\tilde{\beta} \sum A_{i} A_{i}^{\prime}+\sum \lambda_{i}^{\prime} A_{i}+\sum \lambda_{i} A_{i}^{\prime}\right)\right) & = & k .
\end{array}
$$

Call $j^{*}(\tilde{\beta})=\bar{\beta}$. We can write $j^{*}(c)=1+q$, where $q$ is a 4 dimensional cohomology class. So $\bar{\beta}=1+\bar{c}_{1}$ and $q=\bar{c}_{2}$, where $\bar{c}=1+\bar{c}_{1}+\ldots$. From the above identities we get $\mu_{i}=\lambda_{i}$ and $\mu_{i}^{\prime}=\lambda_{i}^{\prime}$, and

$$
\begin{array}{ll}
\bar{\beta} j^{*}\left(A_{i}\right) & =\left(b_{i}+\lambda_{i}\right) /(1+q)-\lambda_{i}, \\
\bar{\beta} j^{*}\left(A_{i}^{\prime}\right) & =\left(b_{i}^{\prime}+\lambda_{i}^{\prime}\right) /(1+q)-\lambda_{i}^{\prime}, \\
j^{*}\left(\tilde{\beta}^{2} B+\sum \tilde{\beta} A_{i} \tilde{\beta} A_{i}^{\prime}+\sum \lambda_{i}^{\prime} \tilde{\beta} A_{i}+\sum \lambda_{i} \tilde{\beta} A_{i}^{\prime}\right) & =k \bar{\beta} /(1+q) .
\end{array}
$$

By substituting the first two equalities in the third we see

$$
\begin{aligned}
& \bar{\beta}^{2} j^{*}(B)= k \bar{\beta} /(1+q)-\sum\left[\left(b_{i}+\lambda_{i}\right) /(1+q)-\lambda_{i}\right]\left[\left(b_{i}^{\prime}+\lambda_{i}^{\prime}\right) /(1+q)-\lambda_{i}^{\prime}\right] \\
&-\sum \lambda_{i}^{\prime}\left[\left(b_{i}+\lambda_{i}\right) /(1+q)-\lambda_{i}\right]-\sum \lambda_{i}\left[\left(b_{i}^{\prime}+\lambda_{i}^{\prime}\right) /(1+q)-\lambda_{i}^{\prime}\right] \\
&= k \bar{\beta} /(1+q)-1 /(1+q)^{2} \sum\left(b_{i}+\lambda_{i}\right)\left(b_{i}^{\prime}+\lambda_{i}^{\prime}\right)+\sum \lambda_{i} \lambda_{i}^{\prime} \\
&(1+q)^{2} \bar{\beta}^{2} j^{*}(B)=(1+q) k \bar{\beta}-\sum\left(b_{i}+\lambda_{i}\right)\left(b_{i}^{\prime}+\lambda_{i}^{\prime}\right)+(1+q)^{2} \sum \lambda_{i} \lambda_{i}^{\prime} .
\end{aligned}
$$

By the expression of $B$ above (2), we have

$$
B=k+\sum_{n \geq 1}(-1)^{n+1}(n+1) s_{n}\left(c_{1}(\mathcal{Z}), c_{2}(\mathcal{Z}), \ldots\right)
$$

where $\mathcal{Z} \longrightarrow \tilde{\mathcal{B}}$ is the bundle induced by composing the projection $\pi$ with $Q$ :

$$
\operatorname{Map}^{\bar{P}}(X, B U) \stackrel{\pi}{\longrightarrow} \operatorname{Map}^{k}(X, B S U) \stackrel{Q}{\longrightarrow} B U .
$$

We do not need to identify $\mathcal{Z}$, but according to $[\mathrm{M}] Q$ represents the $K$-theory slant product bundle $\eta_{0} /[X]$. Call $j^{*}(\mathcal{Z})=\zeta$. By expanding both sides, and equating the two dimensional cohomology classes in the last equality (4) we get

$$
\sum\left(b_{i} \lambda_{i}^{\prime}+b_{i}^{\prime} \lambda_{i}\right)=-k \bar{c}_{1}-2 c_{1}(\zeta) .
$$

By equating the four dimensional classes of both sides, and recalling that $q=\bar{c}_{2}$, we get $k \bar{c}_{1}^{2}+2 k \bar{c}_{2}+4 \bar{c}_{1} c_{1}(\zeta)-3 c_{1}(\zeta)^{2}+6 c_{2}(\zeta)=k \bar{c}_{2}-\sum b_{i} b_{i}^{\prime}+2 \bar{c}_{2} \sum \lambda_{i} \lambda_{i}^{\prime}$. Call $e=\left\langle c_{1}(\bar{P})^{2},[X]\right\rangle$. Recall that $k=\left\langle c_{2}(\bar{P}),[X]\right\rangle$, and $e=2 \sum \lambda_{i} \lambda_{i}^{\prime}$. From the last equality

$$
\sum b_{i} b_{i}^{\prime}=(e-k) \bar{c}_{2}-k \bar{c}_{1}^{2}-4 \bar{c}_{1} c_{1}(\zeta)+3 c_{1}(\zeta)^{2}-6 c_{2}(\zeta)
$$

Alternatively, we can derive (5) and (6) by applying Atiyah-Singer's index theorem for families [AS] to $\eta \rightarrow X \times \operatorname{Map}^{\bar{P}}(X, B U(2))$. In our case since the Todd genus $\operatorname{td}(X)=1$ the families of index theorem gives

$$
\operatorname{ch}(\zeta)=\operatorname{ch}\left(j^{*}\left(\eta_{0} /[X]\right)\right)=\operatorname{ch}\left(j^{*}\left(\tilde{\eta} \oplus L^{-1}\right) /[X]\right)=\operatorname{ch}(\eta) /[X]-\operatorname{ch}\left(j^{*} L\right) /[X] .
$$


By plugging the values of the right-hand side from $c\left(j^{*} L\right)=c_{1}(\bar{P}) \times 1+1 \times \bar{c}_{1}$ and from (3) gives also the identities (5) and (6).

The second Chern and the first Pontryagin classes of any $U(2)$-bundle are related by $p_{1}=c_{1}^{2}-4 c_{2}$, and $c(\eta)=1+c_{1}(\eta)+c_{2}(\eta)+\ldots$. Hence

$$
\begin{aligned}
p_{1}(\eta) & =1 \times \bar{p}_{1}+p_{1}(\bar{P}) \times 1+\sum a_{i} \times 2\left(\lambda_{i} \bar{c}_{1}-2 b_{i}\right)+\sum a_{i}^{\prime} \times 2\left(\lambda_{i}^{\prime} \bar{c}_{1}-2 b_{i}^{\prime}\right), \\
\tilde{\Omega} & =2 \sum\left(2 b_{i}^{\prime}-\lambda_{i}^{\prime} \bar{c}_{1}\right)\left(2 b_{i}-\lambda_{i} \bar{c}_{1}\right) .
\end{aligned}
$$

By expanding $\Omega$ and substituting from (5) and (6) above we get

$$
\tilde{\Omega}=8(e-k) \bar{c}_{2}+(e-4 k) \bar{c}_{1}^{2}-24\left[\bar{c}_{1} c_{1}(\zeta)-c_{1}(\zeta)^{2}+2 c_{2}(\zeta)\right] .
$$

By substituting $\bar{c}_{1}^{2}=\bar{p}_{1}+4 \bar{c}_{2}$ and $e=p_{1}+4 k$ we get

$$
\tilde{\Omega}=p_{1} \bar{p}_{1}+12\left(p_{1}+2 k\right) \bar{c}_{2}-24\left[\bar{c}_{1} c_{1}(\zeta)-c_{1}(\zeta)^{2}+2 c_{2}(\zeta)\right] .
$$

We can also prove a version of Proposition 2.2 when $X$ is odd: We can find a degree 2 map from an even manifold $F: Y \rightarrow X$, and by naturality properties of $\Omega$ we get $F^{*}(\Omega(Y))=4 \Omega(X)$.

Corollary 2.3. If $X$ is odd, then $4 \Omega-p_{1} \bar{p}_{1}=0 \bmod 6$. If furthermore $\pi_{1}\left(\mathcal{B}^{*}\right)=0$ then $4 \Omega-p_{1} \bar{p}_{1}=0 \bmod 12$, in particular, if $X$ is odd then $p_{1} \bar{p}_{1}=0 \bmod 4$.

Since $\pi_{1}\left(\mathcal{B}^{*}\right)$ is 0 or $\mathbf{Z}_{2}$, torsion subgroup of $H^{2}\left(\mathcal{B}^{*}\right)$ is 0 or $\mathbf{Z}_{2}$. Formula (5) of the above proof gives a result of [D]; namely if $P \rightarrow X$ is an $S U(2)$-bundle (i.e. $\lambda_{i}$ and $\left.\lambda_{i}^{\prime}=0\right)$ with $c_{2}(P)=k$ odd, then the universal fibration $\xi \rightarrow X \times \mathcal{B}^{*}$ lifts to a $S U(2)$-bundle (i.e. $\bar{c}_{1}$ is even). Also notice that $c_{2}(P)$ odd implies $\pi_{1}\left(\mathcal{B}^{*}\right)=0$ (Proposition 4.1).

In case of $S U(2)$-bundles the fibration $K\left(\mathbf{Z}_{2}, 1\right) \rightarrow B S U(2) \rightarrow B S O(3)$ induces the fibration

$$
K\left(\mathbf{Z}_{2}, 1\right) \rightarrow \operatorname{Map}(X, B S U(2)) \stackrel{\pi}{\longrightarrow} \operatorname{Map}(X, B S O(3)) .
$$

By $[\mathrm{M}] \pi^{*}\left(p_{1} \bar{p}_{1}+2 m \Omega\right) \bar{p}_{1}^{m-1} \in H^{4 m}(\operatorname{Map}(X, B S U(2)))$ is divisible by $2 m+1 . \pi$ induces isomorphism in cohomology groups with $\mathbf{Z}_{2 m+1}$ coefficients hence:

Proposition 2.4. If $P \rightarrow X$ is an $S U(2)$-bundle, then the class $\left(\Omega-p_{1} \bar{p}_{1}\right) \bar{p}_{1}^{m-1}$ in $H^{4 m}\left(\mathcal{B}^{*}\right)$ is divisible by $2 m+1$.

\section{Postnikov tower of $B U(2)$ And the Associated COHOMOLOGY OPERATIONS}

Computation of the homotopy groups of $\mathcal{B}^{*}(P)$ can be reduced to the problem counting number of $U(2)$-bundles and extending $U(2)$-bundles on associated spaces. Postnikov tower of $B U(2)$ is most suited for this type of problem. It consists of maps and spaces $B U(2) \rightarrow \ldots \rightarrow X_{n+1} \rightarrow X_{n} \rightarrow \ldots \rightarrow X_{1}$ and cohomology classes $k_{n+2} \in H^{n+2}\left(X_{n} ; \pi_{n+1}\right)$, where $\pi_{n}=\pi_{n} B U(2)$. Existence of an $U(2)$-bundle over the $(n+1)$-skeleton of a space $Z$ is equivalent to having a map $f_{n}: Z \rightarrow X_{n}$, and extending this bundle to an $(n+2)$-skeleton (after readjusting over the $(n+1)$ cells) is equivalent to showing $f_{n}^{*}\left(k_{n+2}\right)=0$. Each $X_{n+2} \rightarrow X_{n}$ is a fibration with fiber $K\left(\pi_{n+1}, n+1\right)$, obtained by pulling back the universal fibration by the map $k_{n+2}: X_{n} \rightarrow K\left(\pi_{n+1}, n+2\right)$. In particular the fundamental cohomology class of the fiber transgresses to $k_{n+2}$. At the end of this section we will compute some useful secondary cohomology operations induced by the Postnikov tower of $B U(2)$. 
Proposition 3.1. The 6 stage Postnikov tower of $B U(2)$ is given by :

$$
\begin{array}{rll}
K\left(\mathbf{Z}_{2}, 6\right) \stackrel{j}{\longrightarrow} X_{6} & \stackrel{k_{8}^{1} \times k_{8}^{2}}{\longrightarrow} & K\left(\mathbf{Z}_{4}, 8\right) \times K\left(\mathbf{Z}_{3}, 8\right) \\
\pi_{5} \downarrow & & \\
K\left(\mathbf{Z}_{2}, 5\right) \stackrel{j}{\longrightarrow} X_{5} & \stackrel{k_{7}}{\longrightarrow} & K\left(\mathbf{Z}_{2}, 7\right) \\
\pi_{4} \downarrow & & \\
B U(2) \stackrel{f}{\longrightarrow} X_{4} & \stackrel{k_{6}}{\longrightarrow} & K\left(\mathbf{Z}_{2}, 6\right)
\end{array}
$$

where $X_{4}=K(\mathbf{Z}, 2) \times K(\mathbf{Z}, 4)$, and $f=\left(c_{1}, c_{2}\right)$ is given by the Chern classes. Also if $i_{n}$ denotes the corresponding fundamental classes of $H^{n}\left(K(\mathbf{Z}, n): \mathbf{Z}_{2}\right)$ and $H^{n}\left(K\left(\mathbf{Z}_{2}, n\right): \mathbf{Z}_{2}\right)$, and $S q^{k}, S t_{3}^{k}$ are the $k$-Steenrod square and cube operations, and $\rho_{2}: K\left(\mathbf{Z}_{4}, 8\right) \rightarrow K\left(\mathbf{Z}_{2}, 8\right)$ is the $\bmod 2$ reduction then:

$$
\begin{aligned}
& k_{6}=S q^{2} i_{4}+i_{2} i_{4}, \\
& j \circ k_{7}=S q^{2}\left(i_{5}\right), \\
& \rho_{2} \circ k_{8}^{1}=r \circ \pi_{5}, \text { where } r: X_{5} \rightarrow K\left(\mathbf{Z}_{2}, 8\right) \text { with } r \circ j=S q^{2} S q^{1}\left(i_{5}\right), \\
& k_{8}^{2}=s \circ \pi_{4} \circ \pi_{5}, \text { where } s: X_{4} \rightarrow K\left(\mathbf{Z}_{3}, 8\right) \text { with } s=S t_{3}^{1}\left(i_{4}\right)-i_{4} i_{2}^{2}-i_{4}^{2} .
\end{aligned}
$$

Proof. The 4-skeleton of $B U(2)$ is $K(\mathbf{Z}, 2) \times K(\mathbf{Z}, 4)$ and the map $f=\left(c_{1}, c_{2}\right)$ : $B U(2) \rightarrow K(\mathbf{Z}, 2) \times K(\mathbf{Z}, 4)$ induces isomorphism on homotopy up to and including dimension 4. If $F_{5}$ is the fiber of the map $f$, then $\pi_{k}\left(F_{5}\right)=0$ for all $k \leq 4$ and $\pi_{5}\left(F_{5}\right)=\pi_{5}(B S U(2))=\mathbf{Z}_{2}$. By the Serre exact sequence the first $k$-invariant $k_{6}$ is the image of the fundamental class under transgression $\tau$

$$
\ldots \longrightarrow H^{5}\left(F_{5} ; \mathbf{Z}_{2}\right) \stackrel{\tau}{\longrightarrow} H^{6}\left(X_{4} ; \mathbf{Z}_{2}\right) \stackrel{f^{*}}{\longrightarrow} H^{6}\left(B U(2) ; \mathbf{Z}_{2}\right) .
$$

So $k_{6}$ is the unique element in $H^{6}\left(X_{4} ; \mathbf{Z}_{2}\right)$ with $f^{*}\left(k_{6}\right)=0$. We claim that $k_{6}=$ $S q^{2} i_{4}+i_{2} i_{4}$. To check this we need to show that $f^{*}\left(k_{6}\right)=S q^{2} c_{2}+c_{1} c_{2}$ vanishes. By the splitting principle we can assume that the universal bundle is a direct sum of line bundles $L_{1} \oplus L_{2}$ hence

$$
\begin{aligned}
c_{1} & =c_{1} L_{1}+c_{1} L_{2}, \quad c_{2}=c_{1} L_{1} \cdot c_{1} L_{2}, \\
S q^{2} c_{2}+c_{1} c_{2} & =2\left(\left(c_{1} L_{1}\right)^{2} \cdot c_{1} L_{2}+c_{1} L_{1} \cdot\left(c_{1} L_{2}\right)^{2}\right)=0 \quad \bmod 2 .
\end{aligned}
$$

Let $K\left(\mathbf{Z}_{2}, 5\right) \stackrel{j}{\longrightarrow} X_{5} \stackrel{\pi_{4}}{\longrightarrow} X_{4}$ be the induced fibration by $k_{6}$ from the universal fibration over $K\left(\mathbf{Z}_{2}, 6\right)$. By construction the fundamental class of $K\left(\mathbf{Z}_{2}, 5\right)$ transgresses to $k_{6}$, and $f$ lifts to a map $f_{5} ; B U(2) \rightarrow X_{5}$. The fiber $F_{6}$ of this map $f_{5}$ is 5 -connected, and $\pi_{6}\left(F_{6}\right)=\pi_{6} B U(2)=\mathbf{Z}_{2}$. The transgression of the fundamental class of $H^{6}\left(F_{5} ; \mathbf{Z}_{2}\right)$ is the next $k$-invariant $k_{7} \in H^{7}\left(X_{5} ; \mathbf{Z}_{2}\right)$. This class has the property $f_{5}^{*}\left(k_{7}\right)=0$ in fact by the Serre exact sequence

$$
\ldots \longrightarrow H^{6}\left(F_{6} ; \mathbf{Z}_{2}\right) \stackrel{\tau}{\longrightarrow} H^{7}\left(X_{5} ; \mathbf{Z}_{2}\right) \stackrel{f_{5}^{*}}{\longrightarrow} H^{7}\left(B U(2) ; \mathbf{Z}_{2}\right)=0 .
$$

By using the spectral sequence of the fibration $K\left(\mathbf{Z}_{2}, 5\right) \rightarrow X_{5} \rightarrow X_{4}$ we see that $k_{7}$ is the class as described in the statement of the theorem:

$$
E_{2}^{p, q}=H^{p}\left(X_{4} ; \mathbf{Z}_{2}\right) \otimes H^{q}\left(K\left(\mathbf{Z}_{2}, 5\right) ; \mathbf{Z}_{2}\right) .
$$

Let us denote the two and four dimensional fundamental classes of $X_{4}$ by $a$ and $b$, respectively. Since these come from integral classes $S q^{1}(a)=S q^{1}(b)=0$. By the spectral sequence the only classes that can possibly contribute to the seven dimensional cohomology of $X_{5}$ are: $S q^{2}\left(i_{5}\right), a \otimes i_{5}$, and the seven dimensional class $S q^{3}(b)$ of $X_{4}$. By the transgression relation $d_{6}\left(i_{5}\right)=k_{6}=S q^{2}(b)+a b$, we have

$$
d_{7}\left(S q^{1}\left(i_{5}\right)\right)=S q^{1}\left(k_{6}\right)=S q^{1} S q^{2}(b)=S q^{3}(b)
$$




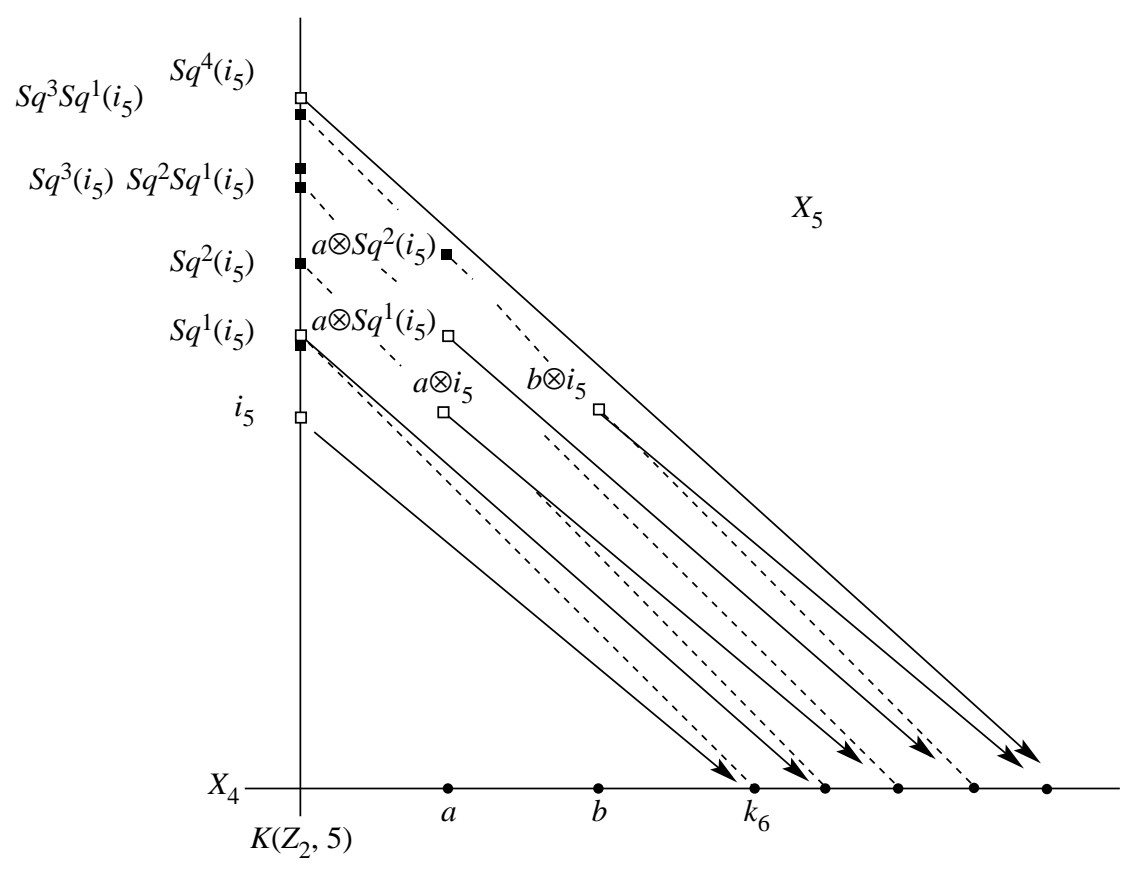

Figure 2

also

$$
d_{6}\left(a \otimes i_{5}\right)=a k_{6}=a S q^{2}(b)+a^{2} b \neq 0
$$

Hence $S q^{2}\left(i_{5}\right)$ is the only possible class that could contribute to the homology, and in fact this is the case since

$$
\begin{aligned}
d_{8}\left(S q^{2}\left(i_{5}\right)\right) & =S q^{2}\left(k_{6}\right)=S q^{2}\left(S q^{2}(b)+a b\right)=S q^{2} S q^{2}(b)+a^{2} b+a S q^{2}(b) \\
& =S q^{3} S q^{1}(b)+a^{2} b+a S q^{2}(b)=a^{2} b+a S q^{2}(b)
\end{aligned}
$$

and the last element has already been killed by $d_{6}\left(a \otimes i_{5}\right)$. So, $S q^{2}\left(k_{6}\right)$ survives to give the only homology class in $H^{7}\left(X_{5} ; \mathbf{Z}_{2}\right)$ and this must be the class $k_{7}$ by the above exact sequence. From this spectral sequence, by a routine calculation, we also get that $H^{8}\left(X_{5} ; \mathbf{Z}_{2}\right)=\mathbf{Z}_{2} \oplus \mathbf{Z}_{2} \oplus \mathbf{Z}_{2} \oplus \mathbf{Z}_{2} \oplus \mathbf{Z}_{2}$ and is generated by $\left\{a^{2} b, a^{4}, b^{2}, \alpha, \beta\right\}$ with the properties: $j^{*}(\alpha)=S q^{3}\left(i_{5}\right)$, and $j^{*}(\beta)=S q^{2} S q^{1}\left(i_{5}\right)$. Furthermore $H^{9}\left(X_{5} ; \mathbf{Z}_{2}\right)=\mathbf{Z}_{2} \oplus \mathbf{Z}_{2}$ and is generated by $\left\{\gamma, a k_{7}\right\}$, such that $j^{*}(\gamma)=S q^{3} S q^{1}\left(i_{5}\right)$. In Figure 2 we indicate the nonzero differentials in the spectral sequence.

Finally, we iterate this process for the last time by taking the fibration $K\left(\mathbf{Z}_{2}, 6\right)$ $\rightarrow X_{6} \stackrel{\pi_{5}}{\longrightarrow} X_{5}$ induced from $K\left(\mathbf{Z}_{2}, 7\right)$ by $k_{7}$. Again, the fundamental class of $K\left(\mathbf{Z}_{2}, 6\right)$ transgresses to $k_{7}$, and $f_{5}$ lifts to $f_{6}: B U(2) \rightarrow X_{6}$. The 6-connected fiber $F_{7}$ of $f_{6}$ has the first homotopy $\pi_{7}\left(F_{7}\right)=\pi_{7} B U(2)=\pi_{6}\left(S^{3}\right)=\mathbf{Z}_{12}$. Then $k_{8} \in H^{8}\left(X_{6} ; \mathbf{Z}_{12}\right)$ is the image of the fundamental class under the transgression, and it has the property $f_{6}^{*}\left(k_{8}\right)=0$. Since $H^{8}\left(X_{6} ; \mathbf{Z}_{12}\right)=H^{8}\left(X_{6} ; \mathbf{Z}_{4}\right) \oplus H^{8}\left(X_{6} ; \mathbf{Z}_{3}\right)$ we can decompose $k_{8}=\left(k_{8}^{1}, k_{8}^{2}\right)$. Let us first compute $k_{8}^{1}$. To do this we first compute $H^{8}\left(X_{6} ; \mathbf{Z}_{2}\right)$ by using the spectral sequence of the fibration $K\left(\mathbf{Z}_{2}, 6\right) \rightarrow X_{6} \stackrel{\pi_{5}}{\longrightarrow} X_{5}$. $E_{2}^{p, q}=H^{p}\left(X_{5} ; \mathbf{Z}_{2}\right) \otimes H^{q}\left(K\left(\mathbf{Z}_{2}, 6\right) ; \mathbf{Z}_{2}\right)$. So, the only possible elements that could give the eight dimensional homology of $X_{6}$ are: $S q^{2}\left(i_{6}\right), a \otimes i_{6}$, and the eight 


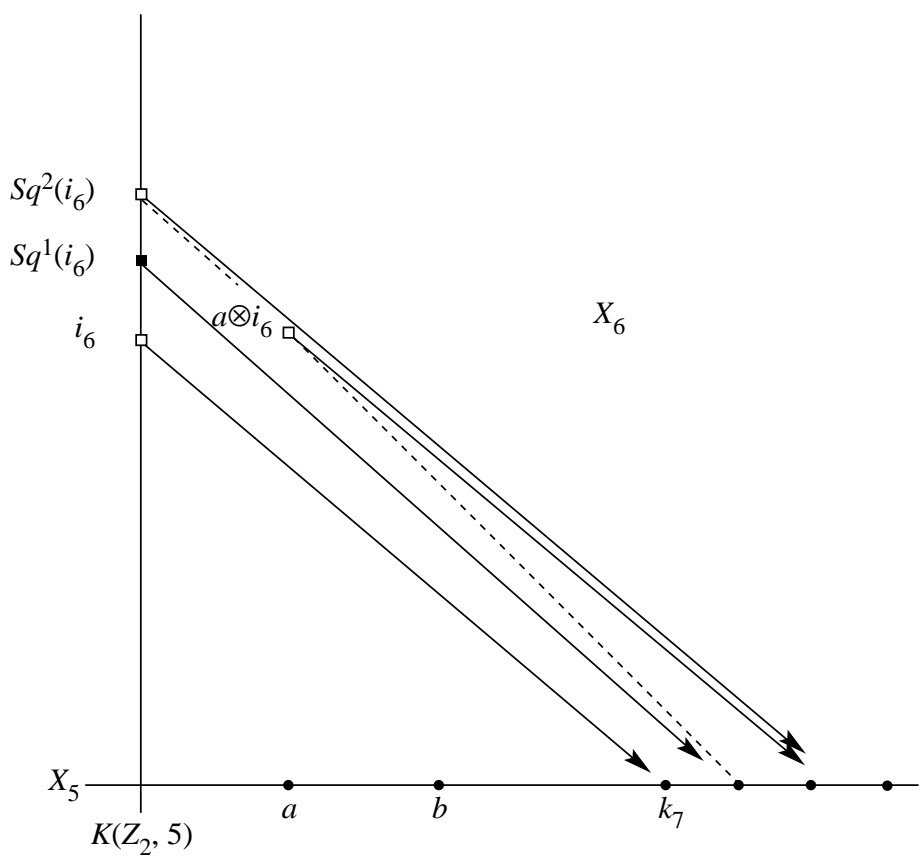

FiguRE 3

dimensional classes $\left\{a^{2} b, a^{4}, b^{2}, \alpha, \beta\right\}$ of $X_{5}$. Since $d_{7}\left(i_{6}\right)=k_{7}$ we have $d_{7}\left(a \otimes i_{6}\right)=$ $a k_{7} \neq 0, a \otimes i_{6}$ can not contribute. Since $d_{9}\left(S q^{2}\left(i_{6}\right)\right)=S q^{2}\left(k_{7}\right)$ and $j^{*}\left(S q^{2}\left(k_{7}\right)\right)=$ $S q^{2}\left(j^{*}\left(k_{7}\right)\right)=S q^{2} S q^{2}\left(i_{5}\right)=S q^{3} S q^{1}\left(i_{5}\right)$, from the above calculation, we see that $S q^{2}\left(k_{7}\right)$ must be a nontrivial element of $H^{9}\left(X_{5} ; \mathbf{Z}_{2}\right)$. It is also easy to check that this element $S q^{2}\left(k_{7}\right)$ has not been killed by the previous differentials, hence $S q^{2}\left(i_{6}\right)$ does not survive the given nontrivial cohomology class. Also $d_{8}\left(S q^{1}\left(i_{6}\right)\right)=S q^{1}\left(k_{7}\right)$, and $j^{*}\left(S q^{1}\left(k_{7}\right)\right)=S q^{1}\left(j^{*} k_{7}\right)=S q^{1} S q^{2}\left(i_{5}\right)=S q^{3}\left(i_{5}\right)$ by the above calculation. $S q^{1}\left(k_{7}\right)$ is equal to $\alpha$ up to an addition with classes $\left\{a^{2} b, a^{4}, b^{2}\right\}$. After a change of basis we can assume $S q^{1}\left(k_{7}\right)=\alpha$. So, $\alpha$ does not survive the spectral sequence. After a routine check it is readily seen that the remaining classes do survive to give the homology of $X_{6}$. So $H^{8}\left(X_{6} ; \mathbf{Z}_{2}\right)=\mathbf{Z}_{2} \oplus \mathbf{Z}_{2} \oplus \mathbf{Z}_{2} \oplus \mathbf{Z}_{2}$, and it is generated by $\left\{a^{2} b, a^{4}, b^{2}, C\right\}$, such that $C=\pi_{5}^{*}(\beta)$. Figure 3 shows the nonzero differentials.

Now notice that

$$
\begin{aligned}
j^{*} S q^{1}(\beta) & =S q^{1} j^{*}(\beta)=S q^{1} S q^{2} S q^{1}\left(i_{5}\right)=S q^{3} S q^{1}\left(i_{5}\right) \\
& =S q^{2} S q^{2}\left(i_{5}\right)=S q^{2} j^{*}\left(k_{7}\right)=j^{*} S q^{2}\left(k_{7}\right) .
\end{aligned}
$$

Hence by our previous calculations $S q^{1}(\beta)=S q^{2}\left(k_{7}\right)=\gamma$ then

$$
S q^{1}(C)=S q^{1} \pi_{5}^{*}(\beta)=\pi_{5}^{*} S q^{1}(\beta)=\pi_{5}^{*} S q^{2}\left(k_{7}\right)=S q^{2} \pi_{5}^{*}\left(k_{7}\right)=0 .
$$

Therefore $C$ is actually a class with $Z_{4}$ coefficients and it is easily seen that $f_{6}^{*}(C)=$ 0 and $C=k_{8}^{1} \in H^{8}\left(X_{6} ; \mathbf{Z}_{4}\right)$.

Now it remains to compute $k_{8}^{3} \in H^{8}\left(X_{6} ; \mathbf{Z}_{3}\right)$. Since mod 3 cohomology does not see the torsion in $K\left(\mathbf{Z}_{2}, n\right)$ we see that $k_{8}^{3}=\left(\pi_{4} \circ \pi_{5}\right)^{*}(s)$ for some $s \in H^{8}\left(X_{4} ; \mathbf{Z}_{3}\right)$. We must also have $f^{*}(s)=0$ in $\mathbf{Z}_{3}$ cohomology. We can easily see that $s=S t_{3}^{1}\left(i_{2}\right)-$ $i_{2} i_{1}^{2}-i_{2}^{2}$ is the unique element satisfying this property. We can check this (as in 
the case of $k_{4}$ ) by using the splitting principle, i.e. by assuming that the universal bundle is a direct sum of line bundles $L_{1} \oplus L_{2}$. Then $f^{*} s=S t_{3}^{1}\left(c_{2}\right)-c_{2} c_{1}^{2}-c_{2}^{2}$, and the following calculation gives $f^{*} s=0$.

$$
\begin{aligned}
S t_{3}^{1}\left(c_{2}\right) & =S t_{3}^{1}\left(c_{1} L_{1} \cdot c_{1} L_{2}\right)=S t_{3}^{1}\left(c_{1} L_{1}\right) \cdot c_{1} L_{2}+c_{1} L_{1} \cdot S t_{3}^{1}\left(c_{1} L_{2}\right) \\
& =\left(c_{1} L_{1}\right)^{3} \cdot c_{1} L_{2}+c_{1} L_{1} \cdot\left(c_{1} L_{2}\right)^{3}=c_{1} L_{1} \cdot c_{1} L_{2}\left(\left(c_{1} L_{1}\right)^{2}+\left(c_{1} L_{2}\right)^{2}\right) \\
& =c_{2}\left(\left(c_{1} L_{1}+c_{1} L_{2}\right)^{2}-2 c_{1} L_{1} \cdot c_{1} L_{2}\right)=c_{2}\left(c_{1}^{2}-2 c_{2}\right) \bmod 3 .
\end{aligned}
$$

The $k$-invariants of $B U(2)$ gives rise to secondary cohomology operations. To compute them we need to know how they behave under pull-backs. Let $E \rightarrow B U(2)$ be the bundle obtained by pulling back $X_{5} \rightarrow X_{4}$ by $f$. Since $k_{6} \circ f$ is null homotopic, $E$ can be identified by the trivial bundle

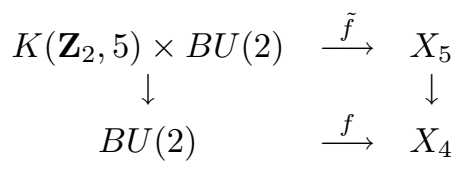

Let $\tilde{f}$ be the map covering $f$, then we have

Lemma 3.2.

$$
\tilde{f}^{*}\left(k_{7}\right)=S q^{2}\left(i_{5}\right) \times 1+i_{5} \times c_{1} .
$$

Proof. First of all by construction $\tilde{f}^{*}\left(k_{7}\right)$ has to be nonzero since it has to restrict to $S q^{2}\left(i_{5}\right)$ on the fiber. By considering $f$ and $\tilde{f}$ as inclusions, we can apply the Thomas exact sequence of this relative fibration $([\mathrm{T}]$, p. 18) to get the exact sequence:

$$
. . \longrightarrow H^{7}\left(X_{5} ; \mathbf{Z}_{2}\right) \stackrel{\tilde{f}^{*}}{\longrightarrow} H^{7}\left(K\left(\mathbf{Z}_{2}, 5\right) \times B U(2) ; \mathbf{Z}_{2}\right) \stackrel{\tau}{\longrightarrow} H^{8}\left(X_{4}, B U(2) ; \mathbf{Z}_{2}\right)
$$

where $\tau$ is the relative transgression. Since $H^{7}(B U(2))=0 \quad \tau$ takes values in $H^{8}\left(X_{4} ; \mathbf{Z}_{2}\right)$. If $g: K\left(\mathbf{Z}_{2}, 5\right) \times B U(2) \rightarrow X_{4}$ is the projection followed by $f$, then we can define a right action of $H^{*}\left(X_{4} ; \mathbf{Z}_{2}\right)$ on $H^{*}\left(K\left(\mathbf{Z}_{2}, 5\right) \times B U(2) ; \mathbf{Z}_{2}\right)$ by $u . v=u \smile g^{*}(v)$. By $([\mathrm{T}]$, p. 14), the transgression is equivariant under this action i.e. $\tau(u . v)=\tau(u) \smile v$. Hence

$$
\begin{array}{r}
\tau\left(i_{5} \times c_{1}\right)=\tau\left(\left(i_{5} \times 1\right) \smile\left(1 \times c_{1}\right)\right)=\tau\left(\left(i_{5} \times 1\right) \smile g^{*}\left(i_{2}\right)\right) \\
=\tau\left(i_{5} \times 1\right) \smile i_{2}=\left(S q^{2}\left(i_{4}\right)+i_{2} i_{4}\right) i_{2}=S q^{2}\left(i_{2} i_{4}\right) .
\end{array}
$$

$i_{2}$ and $i_{4}$, being integral classes, are annihilated by $S q^{1}$. Since $S q^{2} S q^{2}=S q^{3} S q^{1}$ we have $S q^{2} S q^{2}\left(i_{4}\right)=0$. Therefore

$$
\tau\left(S q^{2}\left(i_{5} \times 1\right)\right)=S q^{2}\left(k_{6}\right)=S q^{2}\left(i_{2} i_{4}\right) .
$$

Therefore $S q^{2}\left(i_{5}\right) \times 1+i_{5} \times c_{1}$ lies in the kernel of $\tau$, hence by the exact sequence this must be $\tilde{f}^{*}\left(k_{7}\right)$ (recall that $k_{7}$ is the only nonzero element of $H^{7}\left(X_{5} ; \mathbf{Z}_{2}\right)$ ).

The fibration $K\left(\mathbf{Z}_{2}, 8\right) \longrightarrow K\left(\mathbf{Z}_{4}, 8\right) \stackrel{\rho_{2}}{\longrightarrow} K\left(\mathbf{Z}_{2}, 8\right)$ allows us to break the $\mathbf{Z}_{4^{-}}$ class $k_{8}^{1}$ into two $\mathbf{Z}_{2}$ classes $k_{8}^{11}$, and $k_{8}^{12}$. This means that we first kill the class $k_{8}^{11}:=\rho_{2} \circ k_{8}^{1}$ in tower construction then kill the other $\mathbf{Z}_{2}$ class that appears in the 
next stage. This allows us to decompose the tower as follows:

$$
\begin{array}{rlll}
K\left(\mathbf{Z}_{2}, 8\right) \stackrel{j}{\longrightarrow} X_{8} & \stackrel{k_{8}^{2}}{\longrightarrow} & K\left(\mathbf{Z}_{3}, 8\right) \\
\pi_{7} \downarrow & & \\
K\left(\mathbf{Z}_{2}, 7\right) \stackrel{j}{\longrightarrow} X_{7} & \stackrel{k_{8}^{12}}{\longrightarrow} & K\left(\mathbf{Z}_{2}, 8\right) \\
\pi_{6} \downarrow & & \\
K\left(\mathbf{Z}_{2}, 6\right) \stackrel{j}{\longrightarrow} X_{6} & \stackrel{k_{8}^{11}}{\longrightarrow} & K\left(\mathbf{Z}_{2}, 8\right) \\
\pi_{5} \downarrow & & \\
K\left(\mathbf{Z}_{2}, 5\right) \stackrel{j}{\longrightarrow} X_{5} & \stackrel{k_{7}}{\pi_{4} \downarrow} & K\left(\mathbf{Z}_{2}, 7\right) \\
B U(2) \stackrel{f}{\longrightarrow} X_{4} & \stackrel{k_{6}}{\longrightarrow} & K\left(\mathbf{Z}_{2}, 6\right)
\end{array}
$$

$k_{8}^{11}=r \circ \pi_{5}$, where $r: X_{5} \rightarrow K\left(\mathbf{Z}_{2}, 8\right)$ with $r \circ j=S q^{2} S q^{1}\left(i_{5}\right)$, and $k_{8}^{12} \circ j=S q^{1}\left(i_{7}\right)$, and rest of $k_{6}, k_{7}, k_{8}^{2}$ as in Proposition 3.1.

For a prime $p$, it is easier to construct the $\bmod p$ Postnikov tower of a space $Z$. This means that if $\mathcal{C}_{p}$ is a class of torsion abelian groups such that the order of each element is prime to $p$; we construct the Postnikov tower of $Z$ by using groups $\pi_{n}(Z)_{(p)}=\pi_{n}(Z) \bmod \mathcal{C}_{p}$. This gives a space $Z_{(p)}$ approximating the mod $p$ homotopy type of $Z$ [MT]. Let us apply this to $B U(2)$. We have $\pi_{n+1} B U(2)=$ $\pi_{n}\left(S^{3}\right)$ for $n \geq 2$, and the smallest integer $n$ such that $\pi_{n}\left(S^{3}\right)$ has a nonzero element of order $p$ is $n=2 p$. Also $\pi_{2 p}\left(S^{3}\right)=\mathbf{Z}_{p} \bmod \mathcal{C}_{p}$. So again by calling $X_{4}=K(\mathbf{Z}, 2) \times K(\mathbf{Z}, 4)$ the first stage of $\bmod p$ resolution looks like

$$
B U(2)_{(p)} \rightarrow X_{4} \stackrel{k_{2 p+2}}{\longrightarrow} K\left(\mathbf{Z}_{p}, 2 p+2\right) .
$$

We claim: $k_{2 p+2}=S t_{p}^{1}\left(i_{4}\right)-i_{4} s_{p-1}\left(i_{2}, i_{4}\right)$, where $s_{p-1}$ is the Newton polynomial satisfying $s_{p-1}\left(\sigma_{1}, \sigma_{2}\right)=x_{1}^{p-1}+x_{2}^{p-1}$ with $\sigma_{1}=x_{1}+x_{2}$ and $\sigma_{2}=x_{1} x_{2}$. To see this pull back $i_{2}$ and $i_{4}$, to $c_{1}$ and $c_{2}$. By the splitting principle assume that the universal bundle is the sum of line bundles $L=L_{1} \oplus L_{2}$. Call $x_{i}=c_{1}\left(L_{i}\right)$

$$
S t_{p}^{1}\left(c_{2}\right)=S t_{p}^{1}\left(x_{1} x_{2}\right)=x_{1}^{p} x_{2}+x_{1} x_{2}^{p}=x_{1} x_{2}\left(x_{1}^{p-1}+x_{2}^{p-1}\right)=c_{2} s_{p-1}\left(c_{1}, c_{2}\right) .
$$

Actually $\pi_{2 p+s}\left(S^{3}\right)_{(p)}=0$ for $s<2 p-3[\mathrm{~T}]$. This implies vanishing of some higher $k$-invariants of the $\bmod p$ tower: $k_{i}=0$ for $2 p+2<i \leq 4 p-2$.

\section{4. Номотору оғ $\mathcal{B}^{*}(P)$}

From the weak homotopy equivalence $\mathcal{B}^{*}(P) \simeq \operatorname{Map}^{P}\left(X, B_{S O(3)}\right)$ we get

$$
\begin{aligned}
\pi_{k}\left(\mathcal{B}^{*}\right) & =\left[S^{k}, s_{0} ; \operatorname{Map}^{P}\left(X, B_{S O(3)}\right), P\right] \\
& =\pi_{0} \operatorname{Map}_{*}\left(S^{k}, \operatorname{Map}^{P}\left(X, B_{S O(3)}\right)\right) \\
& =\pi_{0} \operatorname{Map}\left(S^{k} \times X, B_{S O(3)}\right)^{P} \\
& =\left[S^{k} \times X, B_{S O(3)}\right]^{P} .
\end{aligned}
$$

Here $s_{0}$ denotes the base point of $S^{k}$, and the exponential $P$ above means the set of maps that restrict to $P$ on $s_{0} \times X$ and are homotopic to $P$ on each slice $s \times X$ (by viewing view $P$ as a map). Hence the last expression is just the isomorphism class of bundles over $S^{k} \times X$ which restrict to a bundle isomorphic to $P$ on each 
slice and equal to $P$ on a particular slice:

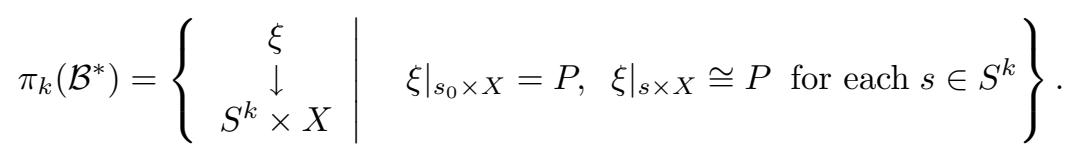

So, $\pi_{k}\left(\mathcal{B}^{*}\right)$ is identified by the number of different bundles on $D^{k} \times X$ restricting to a fixed bundle $P$ on $S^{k-1} \times X$, where $P$ denotes the bundle obtained by pulling back $P$ by the projection to $X$. The group structure is induced by the obvious way of composing two copies of $D^{k} \times X$ along their face $D^{k-1} \times X$.

If $H_{1}(X)=0$ the bundle $P \rightarrow X$ lifts to a $U(2)$-bundle $\bar{P} \rightarrow X$. If case $w_{2}(P)$ is characteristic, i.e. $w_{2}(P)=w_{2}(T X), c_{2}(\bar{P}) \bmod 2$ is a well-defined number, i.e. it is independent of the lifting. Recall by $[\mathrm{AMR}]$ :

\section{Proposition 4.1.}

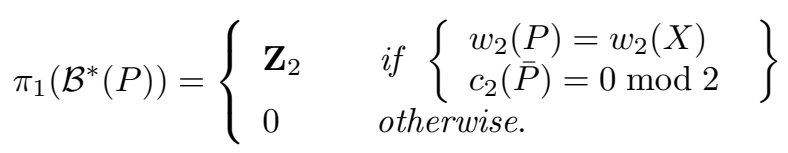

In this section we will compute some higher homotopy groups of $\mathcal{B}^{*}(P)$. Since $D^{k} \times X=S^{k-1} \times X \cup(k-$ cell $) \cup(k+2-$ cells $) \cup(k+4-$ cell $)$, there are no indeterminacies in applying the obstruction theory to count the bundles. For $i=$ $k, k+2, k+4$, let $\left(D^{k} \times X\right)^{(i)}$ denote the $i$-skeleton of $D^{k} \times X$ and

$$
\begin{aligned}
B_{i}= & \text { the number of different bundles on } S^{k-1} \times X \cup\left(D^{k} \times X\right)^{(i)} \\
& \text { restricting to the fixed bundle } P \text { on } S^{k-1} \times X, \\
A_{i}= & \text { the number of different ways to extending a given bundle on } \\
& S^{k-1} \times X \cup\left(D^{k} \times X\right)^{(i-2)} \text { to } S^{k-1} \times X \cup\left(D^{k} \times X\right)^{(i)} .
\end{aligned}
$$

Let $\theta_{i}: B_{i-2} \rightarrow \mathcal{O}_{i}$ be the obstruction to extending to the $i$-skeleton where

$$
\begin{gathered}
A_{i}=H^{i}\left(D^{k} \times X, S^{k-1} \times X ; \pi_{i} B_{S O(3)}\right)=H^{i-k}\left(X ; \pi_{i-1} S O(3)\right), \\
\mathcal{O}_{i}=H^{i}\left(D^{k} \times X, S^{k-1} \times X ; \pi_{i-1} B_{S O(3)}\right)=H^{i-k}\left(X ; \pi_{i-2} S O(3)\right) .
\end{gathered}
$$

For example $\pi_{k}\left(\mathcal{B}^{*}\right)=B_{k+4}$. The group $\pi_{k}\left(\mathcal{B}^{*}\right)$ contains at least the "trivial" element $P$. $B_{k+4}$ is a subset of $\bigoplus H^{i-k}\left(X ; \pi_{i-1} S O(3)\right)$. In particular, all $\pi_{k}\left(\mathcal{B}^{*}\right)$ are finite if $k>4$. We can also identify the obstructions with the $k$-invariants in the Postnikov tower of $B U(2)$.

Let $\pi_{k}\left(\mathcal{B}^{*}\right)_{0}$ be the subgroup of bundles in $\pi_{k}\left(\mathcal{B}^{*}\right)$ which are isomorphic to $P$ in the complement of a point in $S^{k} \times X$. Clearly the elements of $\pi_{k}\left(\mathcal{B}^{*}\right)_{0}$ are obtained from $P$ by clutching at the top skeleton by bundles $\eta \longrightarrow S^{k+4}$. Let $\pi_{k}\left(\mathcal{B}^{*}\right)_{1}$ be the cosets of this subgroup, i.e. it is the equivalence class of bundles which differ in the complement of a point, we have a short exact sequence

$$
0 \rightarrow \pi_{k}\left(\mathcal{B}^{*}\right)_{0} \rightarrow \pi_{k}\left(\mathcal{B}^{*}\right) \rightarrow \pi_{k}\left(\mathcal{B}^{*}\right)_{1} \rightarrow 0 .
$$

$\pi_{k}\left(\mathcal{B}^{*}\right)_{1}$ consists of elements of $B_{k+2}$ which are annihilated by $\theta_{k+4}$. Clearly $\pi_{k}\left(\mathcal{B}^{*}\right)$ is obtained from $\pi_{k}\left(\mathcal{B}^{*}\right)_{1}$ by clutching at the top skeleton by bundles $\eta \longrightarrow S^{k+4}$. For $\alpha \in \pi_{k}\left(\mathcal{B}^{*}\right)_{0}$ and the bundle corresponding to $\eta \in \pi_{k+3} S O(3)$, let $\alpha \sharp \eta$ denote the bundle obtained by clutching $\alpha$ with $\eta$ in a chart. So we have the following useful fact. 
Lemma 4.2. If $\pi_{k+3} S O(3)=\mathbf{Z}_{k}$ and $\eta$ is a generator of this group then

$$
\pi_{k}\left(\mathcal{B}^{*}\right)_{0}= \begin{cases}0 & \text { if } P \sharp \eta \cong P, \\ \mathbf{Z}_{k} & \text { if } P \sharp \eta^{i} ¥ P \text { for all } i=1, \ldots, k .\end{cases}
$$

The following idea of Morgan gives us a useful way of expressing $\alpha \sharp \eta \cong \alpha$.

Lemma 4.3. There is an $S O(3)$-bundle $\xi \longrightarrow S^{1} \times S^{k} \times X-\operatorname{int}\left(B^{5+k}\right)$, such that $\left.\xi\right|_{S^{4+k}}=\eta$ and $\left.\xi\right|_{0 \times S^{k} \times X}=\alpha$ if and only if $\alpha \sharp \eta \cong \alpha$.

Proof.

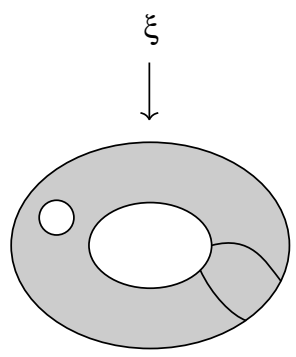

$S^{1} \times S^{1} \times X-\operatorname{int}\left(B^{6}\right)$

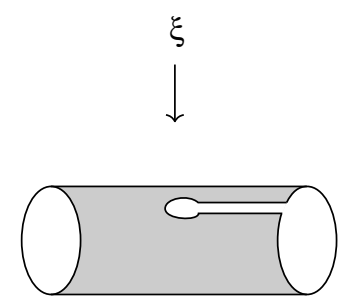

$I \times S^{1} \times X$

FiguRE 4

Given such a bundle $\xi$ we cut $S^{1} \times S^{k} \times X-\operatorname{int}\left(B^{5+k}\right)$ open to get a bundle over the punctured $I \times S^{k} \times X$ which restricts to $\alpha$ on the two outside boundary components and to $\eta$ over the puncture $S^{5}$.

Then by connecting summing $S^{4+k}$ to one of the outside boundary components we get a bundle over $I \times X$ restricting $\alpha$ and $\alpha \sharp \eta$ over the two ends, hence these two bundles are isomorphic. Clearly the converse also holds.

Proposition 4.4. If $\pi_{1}\left(\mathcal{B}^{*}(P)\right)=0$, then

$$
\pi_{2}\left(\mathcal{B}^{*}(P)\right)= \begin{cases}H^{2}(X ; \mathbf{Z}) & \text { if } \quad w_{2}(P) \neq w_{2}(T X), \\ H^{2}(X ; \mathbf{Z}) \oplus \mathbf{Z}_{2} \quad \text { if } \quad w_{2}(P)=w_{2}(T X) .\end{cases}
$$

Proof. We can always assume that $c_{2}(\bar{P})$ is odd. This is because $c_{2}=\frac{1}{4}\left(c_{1}^{2}-p_{1}\right)$ and, hence the changing of the lifting $c_{1}$ to $c_{1}+2 \theta$ changes $c_{2}$ to $c_{2}+\left(c_{1} \theta+\theta^{2}\right)$. So in case $w_{2}(P) \neq w_{2}(T X)$, by choosing $\theta$ we can change the parity of $c_{2}$. When $w_{2}(P)=w_{2}(T X)$ we must already have $c_{2}$ odd, otherwise $\mathcal{B}^{*}(P)$ would not be simply connected by Proposition 4.1.

Let $\xi \longrightarrow S^{2} \times X$ be an element of $\pi_{2}\left(\mathcal{B}^{*}\right)$. Since the complement of a point in $S^{2} \times X$ is a 4 complex, over $S^{2} \times X-\operatorname{int}\left(B^{6}\right)$ this bundle is determined by its first Pontryagin class $p_{1}(\xi)$ and the second Steifel-Whitney class $w_{2}(\xi)$ ([DW]). Since $S^{2} \times X$ is simply connected $\xi$ lifts to a $U(2)$-bundle $\bar{\xi}$. By the Kunneth theorem the Chern classes of $\bar{\xi}$ can be expressed as

$$
c_{1}(\bar{\xi})=1 \times c_{1}(\bar{P})+\epsilon\left(\left[S^{2}\right] \times 1\right), \quad c_{2}(\bar{\xi})=1 \times c_{2}(\bar{P})+\left[S^{2}\right] \times \alpha,
$$

where $\alpha \in H^{2}(X)$ and $\epsilon=0$ or 1 , and $\left[S^{2}\right]$ denotes the fundamental class of $S^{2}$.

$$
\begin{gathered}
w_{2}(\xi)=c_{1}(\bar{\xi}) \bmod 2 \\
p_{1}(\xi)=c_{1}(\bar{\xi})^{2}-4 c_{2}(\bar{\xi})=1 \times p_{1}(P)+\left[S^{2}\right] \times\left(2 \epsilon c_{1}(\bar{P})-4 \alpha\right) .
\end{gathered}
$$


Since $\xi$ is defined on all of $S^{2} \times X$ the obstruction to extending to the top skeleton must vanish (notice if it extends as an $S O(3)$-bundle then it must extend as a $U(2)$ bundle), i.e. $\theta_{6}(\xi)=0$. By definition $\theta_{6}(\xi)$ can be identified with the $k$-invariant $k_{6}$ in the Postnikov tower of $B U(2)$, so

$$
k_{6}(\bar{\xi})=S q^{2}\left(c_{2}(\bar{\xi})\right)+c_{1}(\bar{\xi}) c_{2}(\bar{\xi}) \bmod 2 .
$$

An easy calculation shows that $k_{6}(\bar{\xi})=\alpha^{2}+c_{1} \alpha+\epsilon c_{2} \bmod 2$. Since $c_{2}$ is odd, the condition $k_{6}(\xi)=0$ implies $\epsilon=\left\langle\alpha^{2}+c_{1} \alpha,[X]\right\rangle \bmod 2$, i.e. $\epsilon$ is determined by $\alpha$. Hence $\xi \mid S^{2} \times X-\operatorname{int}\left(B^{6}\right)$ is determined by $\alpha \in H^{2}(X)$. Conversely, given $\alpha$ we can construct the corresponding bundle, since the obstructions to constructing it lies in $\mathcal{O}_{i}=0$ for $i=2,4$. So $\pi_{2}\left(\mathcal{B}^{*}\right)_{1}=H^{2}(X ; \mathbf{Z})$.

We claim that $\pi_{2}\left(\mathcal{B}^{*}\right)_{0}=\mathbf{Z}_{2}$ or 0 according to the $w_{2}(P)$ characteristic or not. Since $\pi_{5} S O(3)=\mathbf{Z}_{2}$. By Lemmas 4.2 and 4.3 it is enough to show that:

(a) Every $S O(3)$-bundle $\eta \longrightarrow S^{1} \times S^{2} \times X-\operatorname{int}\left(B^{7}\right)$ restricting to $\xi$ on $0 \times S^{2} \times X$, extends to $S^{1} \times S^{2} \times X$, when $w_{2}(P)$ is characteristic.

(b) There is an $S O(3)$-bundle $\eta \longrightarrow S^{1} \times S^{2} \times X-\operatorname{int}\left(B^{7}\right)$ which restricts to $\xi$ on $0 \times S^{2} \times X$ and $\left.\eta\right|_{S^{6}}$ is nontrivial, when $w_{2}(P)$ is not characteristic.

Since $S O(3)$-bundles in question all lift to $U(2)$-bundles, it suffices to show (a) and (b) for $U(2)$-bundles. Let $\bar{\xi} \longrightarrow S^{1} \times S^{2} \times X$ also denote the pull-back of $\bar{\xi} \longrightarrow S^{2} \times X$ by projection. Consider the Postnikov tower of $B U(2)$ :

$$
\begin{array}{rllll}
K\left(\mathbf{Z}_{2}, 5\right) & = & K\left(\mathbf{Z}_{2}, 5\right) & = & K\left(\mathbf{Z}_{2}, 5\right) \\
i \downarrow & & \downarrow i & & \downarrow i \\
K\left(\mathbf{Z}_{2}, 5\right) \times\left(S^{1} \times S^{2} \times X\right) & \stackrel{1 \times g}{\longrightarrow} & K\left(\mathbf{Z}_{2}, 5\right) \times B U(2) & \stackrel{\tilde{f}}{\longrightarrow} & X_{5} \stackrel{k_{7}}{\longrightarrow} K\left(\mathbf{Z}_{2}, 7\right) \\
s \uparrow \downarrow & & \downarrow & & \downarrow \\
S^{1} \times S^{2} \times X & \stackrel{g}{\longrightarrow} & B U(2) & \stackrel{f}{\longrightarrow} & X_{4} \stackrel{k_{6}}{\longrightarrow} K\left(\mathbf{Z}_{2}, 6\right)
\end{array}
$$

Here $f: B U(2) \rightarrow K(\mathbf{Z}, 2) \times K(\mathbf{Z}, 4)$ is given by $\left(c_{1}, c_{2}\right)$, and $g$ is the classifying map for $\bar{\xi}$. Since $k_{6} \circ f$ is null homotopic, $f$ and $f \circ g$ induces trivial $K\left(\mathbf{Z}_{2}, 5\right)$-bundles over $B U(2)$ and $S^{1} \times S^{2} \times X$ respectively.

In particular $f \circ g$ lifts to $X_{5}$, and the number of different liftings are given by $H^{5}\left(S^{1} \times S^{2} \times X, 0 \times S^{2} \times X ; \mathbf{Z}_{2}\right)=H_{2}\left(S^{2} \times X ; \mathbf{Z}_{2}\right)$. Let $\tilde{f}: K\left(\mathbf{Z}_{2}, 5\right) \times B U(2) \rightarrow X_{5}$ be the map covering $f$. Then the different liftings $h$ of $f \circ g$ can be constructed by $h=\tilde{f} \circ(1 \times g) \circ s$, where $s=(\beta, i d): S^{1} \times S^{2} \times X \rightarrow K\left(\mathbf{Z}_{2}, 5\right) \times\left(S^{1} \times S^{2} \times X\right)$ is a section of the trivial bundle corresponding to a map $\beta: S^{1} \times S^{2} \times X \rightarrow K\left(\mathbf{Z}_{2}, 5\right)$. By Lemma $3.2 \tilde{f}^{*}\left(k_{7}\right)=S q^{2}\left(i_{5}\right)+i_{5} \times c_{1}$, hence

$$
h^{*}\left(k_{7}\right)=S q^{2}(\beta)+\beta \smile c_{1}(\bar{\xi}) \bmod 2 .
$$

In general we can write $\beta=\left[S^{1}\right] \times\left[S^{2}\right] \times a+\delta\left[S^{1}\right] \times 1 \times[X]$ which gives

$$
h^{*}\left(k_{7}\right)=\left[S^{1}\right] \times\left[S^{2}\right] \times\left(a^{2}+a c_{1}(\bar{P})+\delta \epsilon[X]\right) \bmod 2
$$

where $\epsilon=\left\langle\alpha^{2}+c_{1} \alpha,[X]\right\rangle \bmod 2$. Hence if $w_{2}(P)$ is not characteristic we can find the required bundle of (b) by choosing $\beta$ such that $\delta=0$ and $a \in H^{2}(X)$ with $a^{2}+a c_{1}(\bar{P}) \neq 0 \bmod 2$. This means that by modifying $\bar{\xi}$ over the 5 -skeleton we can get a bundle $\eta$ with the desired properties.

If $w_{2}(P)$ is characteristic then clearly we always have $h^{*}\left(k_{7}\right)=0$. This says that the obstruction to extending is always zero, and (a) follows from Lemma 4.9. 
For $\pi_{1}\left(\mathcal{B}^{*}\right)=0$, we have

$$
H^{2}\left(\mathcal{B}^{*} ; \mathbf{Z}\right)=\operatorname{Hom}\left(H_{2}\left(\mathcal{B}^{*}\right), \mathbf{Z}\right)=\operatorname{Hom}\left(\pi_{2}\left(\mathcal{B}^{*}\right) ; \mathbf{Z}\right)=\operatorname{Hom}\left(H^{2}(X), \mathbf{Z}\right) .
$$

Let $\alpha_{1}, \ldots, \alpha_{n}$ be a basis of $H^{2}(X)$, and $\epsilon_{i}=\left\langle\alpha_{i}^{2}+c_{1} \alpha_{i},[X]\right\rangle \bmod 2$. Then we can explicitly describe the map $\mu: H_{2}(X) \rightarrow H^{2}\left(\mathcal{B}^{*}(P)\right)$ :

Lemma 4.5. $\mu(\theta)\left(\alpha_{i}\right)=4\left\langle\alpha_{i}, \theta\right\rangle-2 \epsilon_{i}\left\langle w_{2}(P), \theta\right\rangle$.

Proof. Let $f_{i}: S^{2} \rightarrow \mathcal{B}^{*}(P)$ correspond to $\alpha_{i} \in H^{2}(X)$. If $\xi_{i} \rightarrow S^{2} \times X$ represents $f_{i}$ then $p_{1}\left(\xi_{i}\right)=1 \times p_{1}(P)+\left[S^{2}\right] \times\left(2 \epsilon_{i} c_{1}(P)-4 \alpha_{i}\right)$. Then $\xi_{i}$ is also the pull-back of the universal bundle $\xi$ by $f_{i} \times 1: S^{2} \times X \rightarrow \mathcal{B}^{*}(P) \times X$, hence

$$
\begin{aligned}
\mu(\theta)\left(\alpha_{i}\right) & =-\left\langle p_{1}\left(\left(f_{i} \times 1\right)^{*} \xi\right) / \theta,\left[S^{2}\right]\right\rangle \\
& =-\left\langle p_{1}\left(\xi_{i}\right),\left[S^{2}\right] \times \theta\right\rangle \\
& =4\left\langle\alpha_{i}, \theta\right\rangle-2 \epsilon_{i}\left\langle c_{1}(P), \theta\right\rangle .
\end{aligned}
$$

In particular if $a_{1}, a_{2}, \ldots, a_{n} \in H_{2}(X)$ are the Poincaré duals of $\alpha_{1}, \ldots, \alpha_{n}$ and $\alpha_{i}^{*} \in \operatorname{Hom}\left(H^{2}(X)\right)$ are the vector space duals of $\alpha_{i}$, then we can write

$$
\mu\left(a_{j}\right)=4 \sum_{i}\left\langle\alpha_{i} \smile \alpha_{j},[X]\right\rangle \alpha_{i}^{*}-2\left\langle w_{2}(P), a_{j}\right\rangle \sum_{i} \epsilon_{i} \alpha_{i}^{*}
$$

Remark. In the remaining case of $\pi_{1}\left(\mathcal{B}^{*}(P)\right)=\mathbf{Z}_{2}$ the above result still holds. Since we do not need the result in this generality we leave the proof as an excerise.

Proposition 4.6.

$$
\pi_{3}\left(\mathcal{B}^{*}(P)\right)_{(3)}= \begin{cases}0 & \text { if } \quad p_{1}(P) \neq 0 \bmod 3 \\ \mathbf{Z}_{3} & \text { if } \quad p_{1}(P)=0 \bmod 3\end{cases}
$$

Proof. For $k=3$ the sets $A_{3}=0$ and $A_{5}=H^{2}\left(X ; \mathbf{Z}_{2}\right)$. We are doing our calculations $\bmod \mathcal{C}$, where $\mathcal{C}$ is the class of torsion abelian groups whose orders prime to 3. Hence there is a unique bundle $\xi \longrightarrow S^{3} \times X-\operatorname{int}\left(B^{7}\right)$ restricting to $P$ on $s_{0} \times X$. As in the proof of Proposition 4.4, by identifying the obstruction $\theta_{7}$ to extending $\xi$ to $S^{3} \times X$ by $k_{7}$, which is $0 \bmod \mathcal{C}$. So $\pi_{3}\left(\mathcal{B}^{*}\right)_{1}=0 \bmod \mathcal{C}$.

By Lemmas 4.1 and 4.2 it is enough to show that when $p_{1}(P)=0 \bmod 3$ every $S O$ (3)-bundle $\eta \longrightarrow S^{1} \times S^{3} \times X-\operatorname{int}\left(B^{8}\right)$ with $\eta \mid 0 \times S^{3} \times X=\xi$, extends to $S^{1} \times S^{3} \times X$; and when $p_{1}(P) \neq 0 \bmod 3$ there exists an $S O(3)$-bundle $\eta$ which does not extend.

Notice that $p_{1}(P)=0 \bmod 3$ implies $c_{1}^{2}(P)=c_{2}(P) \quad \bmod 3$. From Postnikov tower of $B U(2)$ the obstructions to extending $\eta$ are given by the $k$-invariants $\left(k_{8}^{1}, k_{8}^{2}\right)$, and $k_{8}^{1}=0 \bmod \mathcal{C}$. By Proposition $3.1 k_{8}^{2}$ is a primary obstruction and

$$
k_{8}^{2}(\eta)=s(\eta)=S t_{3}^{1} c_{2}(\eta)-c_{2}(\eta) c_{1}(\eta)^{2}-c_{2}(\eta)^{2} .
$$

By the Kunneth theorem the characteristic classes of $\eta$ must look like

$$
c_{1}(\eta)=1 \times 1 \times c_{1}(P), \quad c_{2}(\eta)=1 \times 1 \times c_{2}(P)+\lambda\left(\left[S^{1}\right] \times\left[S^{3}\right] \times 1\right)
$$

where $\lambda \in \mathbf{Z}$. Hence $k_{8}^{2}(\eta)=-\lambda\left[S^{1}\right] \times\left[S^{3}\right] \times\left(c_{1}(P)^{2}+2 c_{2}(P)\right)$. But this vanishes $\bmod 3$ when $p_{1}(P)=0 \bmod 3$.

Proposition 4.7. If $\pi_{1}\left(\mathcal{B}^{*}\right)=0$ and $p$ any odd prime, then $\pi_{4}\left(\mathcal{B}^{*}\right)_{(p)}=\mathbf{Z}$. Furthermore if $p>3$

$$
\pi_{k}\left(\mathcal{B}^{*}\right)_{(p)}= \begin{cases}0 & \text { if } \quad 4<k<2 p-3, \\ \mathbf{Z}_{p} \quad \text { if } \quad k=2 p-3 .\end{cases}
$$


The vector space dual of the induced homology class of the generator $\left[S^{4}\right]$ of $\pi_{4}\left(\mathcal{B}^{*}\right)_{(p)}$ is $\mu^{*}(1)=-\bar{p}_{1} / 4$ when $p>3$ or $p=3$ and $p_{1}(P)=0 \bmod 3$, otherwise it is $\frac{1}{3} \mu^{*}(1)$. In particular if $p_{1}(P) \neq 0 \bmod 3$ then $\bar{p}_{1}\left[S^{4}\right]=0 \bmod 3$.

Proof. For simplicity write $\pi_{k}\left(\mathcal{B}^{*}\right)=\pi_{k}\left(\mathcal{B}^{*}\right)_{(p)}$. Since $\pi_{k}\left(S^{3}\right)$ has no $p$-torsion for $k<2 p$ and we are computing only the $p$ primary components, it follows from the definitions that for $p>3: A_{4}=\mathbf{Z}$ for $k=4$, and $A_{k}=A_{k+2}=A_{k+4}=0$ for $4<k<2 p-3$, and $A_{k+4}=\mathbf{Z}_{p}$ for $k=2 p-3$. Also $\mathcal{O}_{k+2}=\mathcal{O}_{k+4}=0$ for $3<k<2 p-2$. Now the statements $\pi_{4}\left(\mathcal{B}^{*}\right)_{(p)}=\mathbf{Z}$ and $\pi_{k}\left(\mathcal{B}^{*}\right)_{(p)}=0$ for $4<k<2 p-3$, follow from obstruction theory definitions.

Furthermore we conclude that $\pi_{2 p-3}\left(\mathcal{B}^{*}\right)=\pi_{2 p-3}\left(\mathcal{B}^{*}\right)_{0}$, i.e. the only possible nontrivial elements are represented by bundles $\eta \rightarrow S^{2 p-3} \times X$ obtained by twisting the "trivial" bundle $P$ at the top cell. As before by the aid of Lemmas 4.2 and 4.3 we will conclude $\pi_{2 p-3}\left(\mathcal{B}^{*}\right)=\mathbf{Z}_{p}$. This follows by proving that every bundle $\xi \rightarrow\left(S^{1} \times S^{2 p-3} \times X\right)_{0}$, which agrees with $P$ on $0 \times S^{2 p-3} \times X$, extends. We show this by writing $c_{1}(\xi)=1 \times 1 \times c_{1}(P)$ and $c_{2}(\xi)=1 \times 1 \times c_{2}(P)$. The relevant $k$-invariant measuring the obstruction to extension is $k_{2 p+2}$. It is computed at the end of section 3 :

$$
k_{2 p+2}(\xi)=S t_{p}^{1}\left(c_{2}\right)-c_{2} s_{p-1}\left(c_{1}, c_{2}\right)=0 .
$$

Hence by Lemma $4.9 \xi$ extends. In case of $p=3$ and $k=4$, we make the same argument, except we have $\mathcal{O}_{8}=\mathbf{Z}_{3}$ and the obstruction to extending the elements $\lambda \in A_{4}=\mathbf{Z}$ to the top skeleton is the following element of $\mathbf{Z}_{3}$ :

$$
\theta_{8}=\lambda\left[S^{4}\right] \times\left(c_{1}(P)^{2}+2 c_{2}(P)\right) .
$$

$\theta$ vanishes when $p_{1}(P)=0 \bmod 3$ or $\lambda=0 \bmod 3$. Hence the generator $\lambda$ of $\pi_{4}\left(\mathcal{B}^{*}\right)$ is identified by 1 or 3 in $\mathbf{Z}$ according to $p_{1}(P)=0 \bmod 3$ or not.

To see the last assertion let $f: S^{4} \rightarrow \mathcal{B}^{*}$ be the generator, this corresponds to a bundle $\eta \rightarrow S^{4} \times X$ such that $c_{1}(\eta)=1 \times c_{1}(P)$ and $c_{2}(\eta)=1 \times c_{2}(P)+\lambda\left(\left[S^{4}\right] \times 1\right)$ with $\lambda \in \mathbf{Z}$, and hence $p_{1}(\eta)=1 \times p_{1}(P)-4 \lambda\left[S^{4}\right] \times 1 . \eta$ is also the pull-back of the universal bundle $\xi$ by $f \times 1: S^{4} \times X \rightarrow \mathcal{B}^{*}(P) \times X$, hence

$$
\left\langle\bar{p}_{1}, f_{*}\left[S^{4}\right]\right\rangle=\left\langle p_{1}(\xi), f_{*}\left[S^{4}\right] \times 1\right\rangle=\left\langle(f \times 1)^{*} p_{1}(\xi),\left[S^{4}\right] \times 1\right\rangle=-4 \lambda .
$$

Remark 4.8. Similar arguments give $\pi_{5}\left(\mathcal{B}^{*}\right)_{(3)}=H^{2}\left(X ; \mathbf{Z}_{3}\right)$, and for $p>3$

$$
\pi_{k}\left(\mathcal{B}^{*}\right)_{(p)}= \begin{cases}H^{2}\left(X, \mathbf{Z}_{p}\right) & \text { if } k=2 p-1, \\ \mathbf{Z}_{p} & \text { if } k=2 p+1, \\ 0 \quad \text { for other } & 2 p-3<k<4 p-6 .\end{cases}
$$

If the obstructions to extending a bundle $\xi$ from $(k-1)$-skeleton to the $k$ skeleton of a CW-complex vanishes, then we can extend $\xi$ to the $k$-skeleton by possibly readjusting it over the $k-1$ cells. The following lemma says that in the cases we are interested in we can extend it without readjusting the bundle.

Lemma 4.9. Let $\xi \rightarrow\left(S^{1} \times S^{n} \times X\right)_{0}$ be an $S O(3)$-bundle over the punctured $S^{1} \times S^{n} \times X$, where $n=2,3$ or $2 p-3$ for an odd prime $p$. For $n=2$ also assume that $w_{2}(\xi)\left(1 \times S^{2} \times 1\right)=0$. Then this bundle extends to $S^{1} \times S^{n} \times X$, if the obstruction $k_{n+5}$ to extending to the top skeleton vanishes.

Proof. Let $Z=S^{1} \times S^{n} \times X$ and $Z_{0}=Z-\operatorname{int}\left(B^{n+5}\right)$. When $k_{n+5}=0$ by obstruction theory $\xi$ can be extended to $Z$, after possibly changing it over the $n+4$ cells. We claim that this readjustment over $n+4$ cells is not necessary, i.e. if $\xi$ does not 


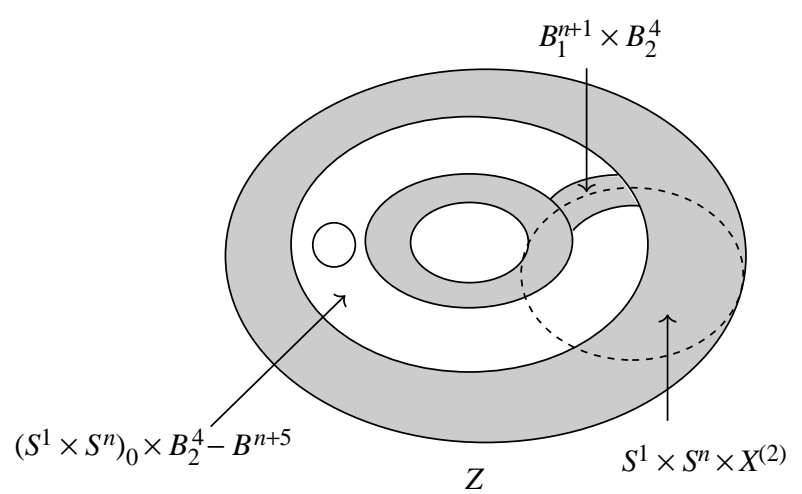

FiguRE 5

extend, then it can not be extended even after readjusting it over the $n+4$ cells. Here we use a trick of $[\mathrm{AMR}]$. If this is not the case we would have two bundles over the complement of the $(n+3)$-skeleton (Figure 5$)$ :

$$
Z_{0}-S^{1} \times S^{n} \times X^{(2)} \cup \operatorname{int}\left(B_{1}^{n+1}\right) \times B_{2}^{4}=\left(S^{1} \times S^{n}\right)_{0} \times B_{2}^{4}-\operatorname{int}\left(B^{n+5}\right)
$$

agreeing over the outside boundary, and one extending the other not extending over $B^{n+5}$. Here $X^{(2)}$ is the two-skeleton of $X, B_{2}^{4}=X-X^{(2)}, B_{1}^{n+1}$ is a ball in $S^{1} \times S^{n}$, and $\left(S^{1} \times S^{n}\right)_{0}$ is the complement of this ball.

By putting these bundles together on the double of this manifold (two copies of the manifold glued along the common boundary) we obtain a bundle on

$$
W=\operatorname{Double}\left(\left(S^{1} \times S^{n}\right)_{0} \times B^{4}\right)-\operatorname{int}\left(B^{n+5}\right)
$$

which is nontrivial on the boundary. $W=S^{1} \times S^{n+4} \sharp S^{n} \times S^{5}-\operatorname{int}\left(B^{n+5}\right)$. Hence we get a map $f: W \rightarrow B_{S O(3)}$ which restricts to the nontrivial element of $\pi_{n+4}\left(B_{S O(3)}\right)=\pi_{n+3}\left(S^{3}\right)$ on the boundary $S^{n+4}$. By surgery we can turn $f$ into a map from a $f: W^{n+5} \rightarrow B_{S O(3)}$ where $W^{n+5}$ is a ball (in case $n=2 p-3$ it is a $\mathbf{Z}_{p}$ homology ball), contradicting the fact that $f$ is essential on $\partial W=S^{n+4}$.

\section{UNIVERSAL RELATIONS}

Let $P \longrightarrow X$ be a $S O(3)$-bundle over a smooth Riemanian 4-manifold $(X, g)$. The moduli space $\mathcal{M}(P)$ of ASD connections on $P$ is defined to be

$$
\mathcal{M}(P)=\left\{[A] \in \mathcal{B}^{*}(P) \mid F_{+}(A)=0\right\}
$$

where $F_{+}(A)$ is the self-dual part of the curvature of the connection $A$ with respect to metric $g$. For generic metrics this is a smooth oriented manifold. For a fixed $w_{2}$, it is customary to call $k=-p_{1}(P) / 4$ and denote $\mathcal{M}_{k}=\mathcal{M}(P)$. In particular, for $S U(2)$-bundles we have $k=c_{2}(P)$. Taubes' gluing construction gives an imbedding representing the gluing parameter:

$$
S O(3) \subset \mathcal{B}^{*}(P)
$$

Proposition 5.1. Let $\gamma \in \pi_{3}\left(\mathcal{B}^{*}(P)\right)$ be the map given by composing the 2-fold covering map with the inclusion $S O(3) \longrightarrow \mathcal{B}^{*}(P)$. Then $\gamma$ represents the generator of $\pi_{3}\left(\mathcal{B}^{*}(P)\right)_{(3)}$. 
Proof. By the exponential principle the homotopy class of the inclusion:

$$
S^{3} \longrightarrow \mathcal{B}^{*}(P)=\operatorname{Map}^{P}\left(X, B_{S O(3)}\right)
$$

describes an $S O(3)$-bundle $\eta \longrightarrow S^{3} \times X$. By [AMR] this bundle is described by the clutching map $\Phi=\pi \circ \phi$, where $\phi: S^{3} \times S^{3} \rightarrow S^{3}, \phi(\rho, x)=x \rho x^{-1}$ and $\pi: S^{3} \rightarrow S O(3)$ is the covering map. Since the restriction of $\phi$ maps $\phi:$ $S^{2} \times S^{3} \rightarrow S^{2}$ it comes from the induced map $\tilde{\phi}: S^{2} * S^{3}=S^{6} \rightarrow S^{3}$. This is the usual Hopf construction applied to $\phi$, and it gives the (Blakers-Massey) generator of $\pi_{6}\left(S^{6}\right)=\mathbf{Z}_{12}$ (e.g. $\left.[\mathrm{J}]\right)$.

Recall that $[\mathrm{DK}] \operatorname{dim}\left(\mathcal{M}_{k}\right)=8 k-3\left(1+b_{2}^{+}\right)$, where $b_{2}^{+}=b_{2}^{+}(X)$ is the dimension of the positive definite part of the intersection form of $X$. In particular when $b_{2}^{+}(X)$ is odd then $\operatorname{dim}\left(\mathcal{M}_{k}\right)=2 d$, for an integer $d:=d_{k}=4 k-\frac{3}{2}\left(1+b_{2}^{+}\right)$.

For $a_{1}, \ldots, a_{d} \in \mathcal{A}_{2}(X)=\left\{a \in H_{2}(X) \mid\left\langle w_{2}(P), a\right\rangle=0\right\}$ the Donaldson invariant is defined to be the intersection number:

$$
\Phi_{d}\left(a_{1}, \ldots, a_{d}\right)=\mathcal{M}_{k} \cap V_{1} \cap V_{2} \cap \ldots \cap V_{d} \in \mathbf{Z}
$$

where $V_{1}, \ldots, V_{d}$ are the codimension two divisors representing the duals of $\mu^{*}\left(a_{1}\right), \ldots$, $\mu^{*}\left(a_{d}\right)$, and

$$
\mu^{*}: \mathcal{A}_{2}(X) \rightarrow H^{2}\left(\mathcal{B}^{*}(P) ; \mathbf{Z}\right)
$$

is the map defined by taking slant product with $-\frac{1}{4} p_{1}(\xi)$ (see section 2 ). In general for $a_{1}, \ldots, a_{d} \in H_{2}(X)$, we take $V_{i}$ to be the divisors corresponding to the integral classes $\mu^{*}\left(2 a_{i}\right)$ and count the intersection number and divide by $2^{d}$. So in general:

$$
\Phi_{d}\left(a_{1}, \ldots, a_{d}\right) \in \mathbf{Z}\left[\frac{1}{2}\right] .
$$

By using the slant product map $\mu^{*}: H_{0}(X) \rightarrow H^{4}\left(\mathcal{B}^{*}(P) ; \mathbf{Z}\right)$ we also have

$$
\Phi_{d}\left(a_{1}, \ldots, a_{d-2}, 1\right)=\left\langle\mu(1), \mathcal{M}_{k} \cap V_{1} \cap V_{2} \cap \ldots \cap V_{d-2}\right\rangle .
$$

Recall that $[\mathrm{KM}]$ a manifold $X$ is called simple if

$$
\Phi_{d+4}\left(a_{1}, \ldots, a_{d}, 1,1\right)=4 \Phi_{d}\left(a_{1}, \ldots, a_{d}\right)
$$

By convention it is usually defined: $\Phi_{d+2}\left(a_{1}, \ldots, a_{d}, 1\right)=2 \Phi_{d}\left(a_{1}, \ldots, a_{d}\right)$.

Now assume $\pi_{1}\left(\mathcal{B}^{*}\right)=0$, then $H^{2}\left(\mathcal{B}^{*}\right)=\operatorname{Hom}\left(H^{2}(X)\right)$. Let $b_{1}, \ldots, b_{n}$ be a basis of $H_{2}(X)$ and $\beta_{1}, \ldots, \beta_{n} \in H^{2}(X)$ be the dual basis with the intersection form $B_{i j}=\left\langle\beta_{i} \smile \beta_{j},[X]\right\rangle$. Then if $\beta_{i}^{*}$ are the vector space duals of $\beta_{i}$ in $\operatorname{Hom}\left(H^{2}(X)\right)$, by Lemma 4.5 and remarks following it we have

$$
\mu\left(2 b_{j}\right)=8 \sum_{r} B_{r j} \beta_{r}^{*} .
$$

From this we can easily calculate that

$$
\Omega=4 \sum B_{r s} \beta_{r}^{*} \smile \beta_{s}^{*} .
$$

We want to compute some homology groups of $\mathcal{B}^{*}(P)$, for this we need to construct its Postnikov tower. We will compute a few stages of the mod $p$ Posnikov tower of $B^{*}$, where $p$ is an odd prime. Let $\mathcal{C}_{p}$ be a class of torsion abelian groups such that the order of each element is prime to $p$. The vector space duals $\left\{\beta_{i}^{*}\right\}$ of $\left\{\beta_{i}\right\}$ determine a map $f: B^{*}(P) \rightarrow Y_{2}$, where $Y_{2}=\prod K(\mathbf{Z}, 2)$ is an $n$-fold product, where $n=\operatorname{rank} H^{2}(X)$. The map $f$ induces isomorphisms on $\pi_{i}$ for $i=1,2$. Let $F_{3}$ be the fiber of $f$. 
Let us first treat the case $p=3$. If $p_{1}(P)=0 \bmod 3$, we have $\pi_{3}\left(B^{*}\right)=\mathbf{Z}_{3}$ $\bmod \mathcal{C}_{p}$ and $H^{3}\left(F_{3} ; \mathbf{Z}_{3}\right)=\mathbf{Z}_{3}$. The first $k$-invariant $k_{4}$ is the transgression of the fundamental class $\tau\left(i_{3}\right)$ in the Serre exact sequence:

$$
\ldots \rightarrow H^{3}\left(F_{3} ; \mathbf{Z}_{3}\right) \stackrel{\tau}{\longrightarrow} H^{4}\left(Y_{2} ; \mathbf{Z}_{3}\right) \stackrel{f^{*}}{\longrightarrow} H^{4}\left(B^{*} ; \mathbf{Z}_{3}\right) \rightarrow \ldots
$$

Hence $k_{4}$ is the unique element in the kernel of $f^{*}$. Since $p_{1}(P)=0 \bmod 3$, by Proposition 2.2 and Corollary 2.3 class $\Omega \in H^{4}\left(B^{*} ; \mathbf{Z}\right)$ is divisible by 3 . We see that $\Omega$ is the pull back of the class $\alpha=\sum_{i j} B_{r s} \gamma_{r} \smile \gamma_{s} \in H^{4}\left(Y_{2}\right)$ by $f$, where $\gamma_{r}=1 \times \ldots \times i_{2} \times \ldots \times 1 \in H^{2}\left(Y_{2}\right)$ are the standard generators (here $i_{2}$ occurring in the $r$ th slot). Since $\left(B_{i j}\right)$ is unimodular intersection form, the $\mathbf{Z}_{3}$-reduction $k_{4} \in H^{4}\left(Y_{2} ; \mathbf{Z}_{3}\right)$ of $\alpha$ is a nonzero class. Also it lies in the kernel of $f^{*}$, where $f^{*}$ is the map induced in cohomology with $\mathbf{Z}_{3}$ coefficients. So $k_{4}$ is the first $k$-invariant. Let $Y_{3}$ be the pull-back fibration by $k_{4}$, and $Y_{4}$ by $k_{5}$ :

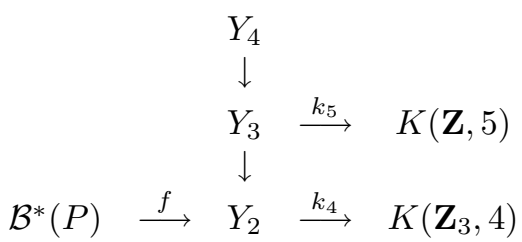

In case $p_{1}(P) \neq 0 \bmod 3$ we have $\pi_{3}\left(B^{*}\right)=0 \bmod \mathcal{C}$. So in this case $k_{4}=0$ and hence $Y_{3}=Y_{2}$. By a simple spectral sequence argument we see that $H^{5}\left(Y_{3}\right)=0$, hence $k_{5}=0$. Therefore the forth stage approximation of $\mathcal{B}_{(3)}^{*}$ is:

$$
Y_{4}= \begin{cases}Y_{3} \times K(\mathbf{Z}, 4) & \text { when } \quad p_{1}(P)=0 \bmod 3 \\ Y_{2} \times K(\mathbf{Z}, 4) & \text { when } \quad p_{1}(P) \neq 0 \bmod 3\end{cases}
$$

Similarly, by using Proposition 4.7 and Remark 4.8 we can construct the Postnikov resolution of $\mathcal{B}_{(p)}^{*}$ for an odd prime $p>3$. From $\pi_{2 p-3}\left(\mathcal{B}^{*}\right)=\mathbf{Z}_{p}$ we get the following nontrivial first stage Postnikov tower of $\mathcal{B}^{*}(P)_{(p)}$ :

$$
\mathcal{B}_{(p)}^{*} \stackrel{F}{\longrightarrow} \prod K(\mathbf{Z}, 2) \times K(\mathbf{Z}, 4) \stackrel{k_{2 p-2}}{\longrightarrow} K\left(\mathbf{Z}_{p}, 2 p-2\right)
$$

where $F=\left(f,-\bar{p}_{1}\right)$. Let $Y_{2 p-4}=\prod K(\mathbf{Z}, 2) \times K(\mathbf{Z}, 4)$. In case $w_{2}(P)=0$, by Proposition 2.4 we can identify $k_{2 p-2}$ as follows: If $\gamma_{i} \in H^{2}\left(Y_{2 p-4} ; \mathbf{Z}_{p}\right), i=1, \ldots, n$, are the two dimensional generators and $\rho \in H^{4}\left(Y_{2 p-4} ; \mathbf{Z}_{p}\right)$ is the four dimensional generator, and $p=2 m+1$ and $p_{1}=\left\langle p_{1}(P),[X]\right\rangle$ then

$$
k_{2 p-2}=4\left(\sum B_{i j} \gamma_{i} \smile \gamma_{j}\right) \smile \rho^{m-1}-p_{1} \rho^{m}
$$

in particular:

$$
F^{*}\left(k_{2 p-2}\right)=\Omega \smile \bar{p}_{1}^{m-1}-p_{1} \bar{p}_{1}^{m}
$$

where $\bar{p}_{1}=-\mu(1)$ is the Pontryagin class of the base point fibration. Recall as an integral class $F^{*}\left(k_{2 p-2}\right)=p \theta_{2 p-2}$, for some $\theta_{2 p-2} \in H^{2 p-2}\left(\mathcal{B}^{*}\right)$.

Let $Y_{2 p-3}$ be the pull-back of the universal fibration by $k_{2 p-2}$ over $Y_{2 p-4}$. Since $\pi_{2 p-2}\left(\mathcal{B}^{*}\right)=0$ the $2 p-2$ stage approximation $Y_{2 p-2}$ of $\mathcal{B}^{*}$ is equal to $Y_{2 p-3}$. The spectral sequence of the fibration $K\left(\mathbf{Z}_{p}, 2 p-3\right) \rightarrow Y_{2 p-3} \rightarrow Y_{2 p-4}$ immediately gives $H^{2 p-2}\left(\mathcal{B}^{*}\right)$ torsion free $\left(\bmod \mathcal{C}_{p}\right)$, and 


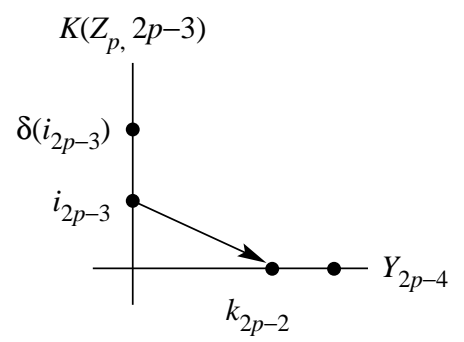

Figure 6

Proposition 5.2. Let $P \rightarrow X$ be an $S U(2)$-bundle, $p=2 m+1$ an odd prime and $k<2 p-2$, then $H^{k}\left(\mathcal{B}^{*} ; \mathbf{Z}_{p}\right)=H^{k}\left(Y_{2 p-4} ; \mathbf{Z}_{p}\right)$. Furthermore assuming $p_{1}(P)=$ $0(\bmod 3)$ in case $p=3$, we have a $\bmod \mathcal{C}_{p}$ short exact sequence:

$$
\begin{gathered}
0 \rightarrow H^{2 p-2}\left(Y_{2 p-4}\right) \stackrel{F^{*}}{\rightarrow} H^{2 p-2}\left(\mathcal{B}^{*}\right) \rightarrow \mathbf{Z}_{p} \rightarrow 0, \\
0 \rightarrow H^{2 p-2}\left(Y_{2 p-4} ; \mathbf{Z}_{p}\right) /\left\langle k_{2 p-2}\right\rangle \stackrel{F^{*}}{\rightarrow} H^{2 p-2}\left(\mathcal{B}^{*} ; \mathbf{Z}_{p}\right) \rightarrow \mathbf{Z}_{p} \rightarrow 0 .
\end{gathered}
$$

In the spectral sequence of $K\left(\mathbf{Z}_{p}, 2 p-3\right) \rightarrow Y_{2 p-3} \rightarrow Y_{2 p-4}$ the fundamental class $i_{2 p-3}$ transgresses to the class $k_{2 p-2}$. So the differential of the mod $p$ Bockstein $\delta\left(i_{2 p-3}\right)$ maps to $\delta\left(k_{2 p-2}\right)$. Since $k_{2 p-2}$ comes from an integral class $\delta\left(k_{2 p-2}\right)=0$, so $\delta\left(i_{2 p-3}\right)$ can be viewed as a class in $H^{2 p-2}\left(Y_{2 p-3} ; \mathbf{Z}_{p}\right)$. In fact if $F: \mathcal{B}^{*} \rightarrow Y_{2 p-3}$ is the approximation map then $F^{*} \delta\left(i_{2 p-3}\right)=\theta_{2 p-2}$. (See Figure 6.)

Let $Y_{k}^{p}$ to be the $k$ th stage Postnikov appoximation of $\mathcal{B}_{(p)}^{*}$. By continuing as above (by using Remark 4.8), we get

$$
\begin{gathered}
Y_{5}^{3}=Y_{4} \times_{k_{6}} \prod K\left(\mathbf{Z}_{3}, 5\right), \\
Y_{4 p-7}^{p}=Y_{2 p-2} \times_{k_{2 p}} \prod K\left(\mathbf{Z}_{p}, 2 p-1\right) \times_{k_{2 p+2}} K\left(\mathbf{Z}_{p}, 2 p+1\right), \quad \text { for } p>3 .
\end{gathered}
$$

The $k$-invariants $k_{2 p}$ and $k_{2 p+2}$ come from classes in $H^{*}\left(Y_{2 p-2} ; \mathbf{Z}_{p}\right)$ and $H^{*}\left(Y_{2 p} ; \mathbf{Z}_{p}\right)$. Similarly, they can be calculated from the divisibility information on classes in $H^{*}\left(\mathcal{B}^{*}\right)$. For example, $k_{2 p}$ is either zero, or else it is given by $\delta\left(i_{2 p-3}\right) \smile \gamma_{i}, i=1,2, \ldots$, or classes in $H^{2 p}\left(Y_{2 p-4}\right)$. To decide which, we need to know whether the classes $\theta_{2 p-2} \smile \beta_{i}^{*}$, or the ones coming from $H^{2 p}\left(Y_{2 p-4}\right)$ in $H^{2 p}\left(\mathcal{B}^{*}\right)$ are divisible by $p$. Notice that vanishing of any of these $k$-invariants creates torsion class in $H^{*}\left(\mathcal{B}^{*}\right)$.

Theorem 5.3. If $P \rightarrow X$ is an $S O(3)$-bundle over a closed smooth simply connected 4-manifold, assume that $c_{2}(P)$ is even when $w_{2}(P)=w_{2}(X)$. Then when $d=2(\bmod 3)$ the following holds: For any basis $b_{1}, \ldots, b_{n}$ of $H_{2}(X)$

$$
\begin{aligned}
& \sum_{i<j} q\left(a_{i}, a_{j}\right) \Phi_{d}\left(a_{1}, \ldots, \widehat{a}_{i}, \ldots, \widehat{a}_{j}, \ldots, a_{d+2}\right) \\
& \quad= \pm \sum_{i, j} \bar{q}\left(b_{i}, b_{j}\right) \Phi_{d+4}\left(a_{1}, \ldots, a_{d+2}, b_{i}, b_{j}\right) \quad(\bmod 3)
\end{aligned}
$$

where $\left(\bar{q}\left(b_{i}, b_{j}\right)\right)$ denotes the inverse of the intersection matrix $\left(q\left(b_{i}, b_{j}\right)\right)$.

Proof. $\operatorname{dim}\left(\mathcal{M}_{k+1}\right)=2 d_{k+1}=\operatorname{dim}\left(\mathcal{M}_{k}\right)+8=2 d_{k}+8$. Choose surfaces $\Sigma_{1}, \ldots$, $\Sigma_{d+2}$, in general position representing $a_{1}, \ldots, a_{d+2}$. Let $V_{i}$ be the corresponding 


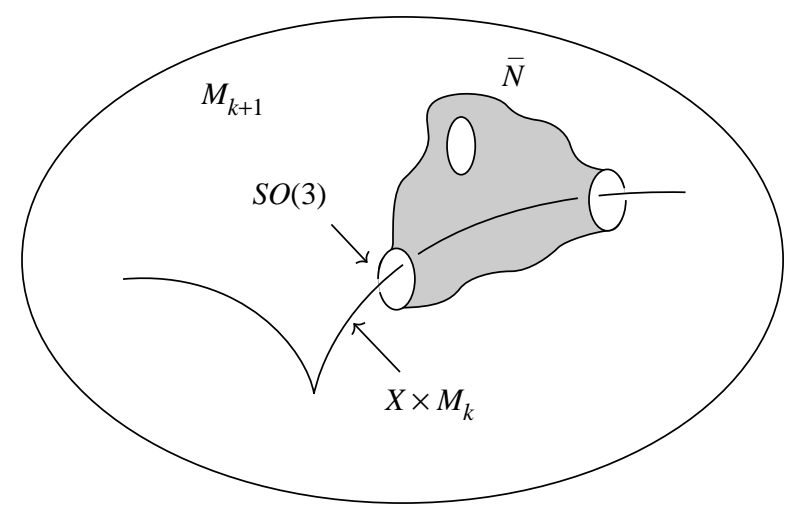

FiguRE 7

divisors that are transverse to each other and to $\mathcal{M}_{k}$ and $\mathcal{M}_{k+1}$. This gives an oriented 4-manifold:

$$
N=V_{1} \cap \cdots \cap V_{d+2} \cap \mathcal{M}_{k+1}
$$

in $\mathcal{B}^{*}\left(P_{k+1}\right)$, where $P_{k+1} \rightarrow X$ is the bundle with $p_{1}\left(P_{k+1}\right)=p_{1}(P)-4$ and $w_{2}\left(P_{k+1}\right)=w_{2}(P)$. Also recall $d_{k}=d$ and $2 d=-2 p_{1}-3\left(1+b_{2}^{+}\right)$. (See Figure 7.)

In general $N$ is not compact. The ends of $N$ compactify to give cones on $S O(3)$. By $[\mathrm{R}]$ each end corresponds to a pair $([A], x)$, where

$$
[A] \in V_{1} \cap \cdots \cap \hat{V}_{i} \cap \cdots \cap \hat{V}_{j} \cap \cdots \cap V_{d+2} \cap \mathcal{M}_{k} \quad \text { and } \quad x \in \Sigma_{i} \cap \Sigma_{j}
$$

and the number of such pairs are measured by the number:

$$
q\left(\Phi_{d}\right)\left(\alpha_{1}, \ldots, \alpha_{d+2}\right):=\sum_{i<j} q\left(a_{i}, a_{j}\right) \Phi_{d}\left(a_{1}, . ., \widehat{a}_{i}, \ldots, \widehat{a}_{j}, \ldots, a_{d+2}\right) .
$$

By cutting the cones we get a compact manifold with boundary $\bar{N} \subset \mathcal{B}^{*}\left(P_{k+1}\right)$, such that each component of the boundary of $\bar{N}$ is the gluing parameter $S O(3)$.

Since $p_{1}(P)=-d=-2 \bmod (3)$, we have $p_{1}\left(P_{k+1}\right)=0 \bmod (3)$. Hence Propositions 4.1 and 4.6 imply $\pi_{1}\left(\mathcal{B}^{*}(P)\right)=0$ and $\pi_{3}\left(\mathcal{B}^{*}(P)\right)_{(3)}=\mathbf{Z}_{3}$. By Proposition 5.1 the two-fold covering map followed by the gluing parameter $S^{3} \rightarrow S O(3) \subset$ $B^{*}(P)$ gives a generator of $\mathbf{Z}_{3}$ in the third homotopy group. So, each boundary component of $\bar{N}$ maps to a fixed generator of $\mathbf{Z}_{3}$. In particular the number $q\left(\Phi_{d}\right)\left(\alpha_{1}, \ldots, \alpha_{d+2}\right) \bmod 3$ represents the $(\bmod 3)$ obstruction to extending the imbedding $\bar{N} \hookrightarrow B^{*}(P)$ to the space $N^{*}$ obtained by coning each boundary component $S O(3)$ of $N$.

We will now compute this obstruction from the mod 3 Postnikov tower of $B^{*}(P)$. Equating these two ways of computing the obstruction will give us the required formula. Since $p_{1}\left(P_{k+1}\right)=0 \bmod 3$, the first $k$-invariant is $k_{4}=\sum_{i j} B_{r s} \gamma_{r} \smile \gamma_{s} \in$ $H^{4}\left(Y_{2} ; \mathbf{Z}_{3}\right)$, where $\left(B_{i j}\right)$ is the intersection form. Since $N$ is orientable and $\mathcal{B}^{*}$ is simply connected, after doing some ambient surgeries in $\mathcal{B}^{*}$, we can assume that it is simply connected. Now it is clear that the only obstruction to extending the inclusion $i: \bar{N} \hookrightarrow \mathcal{B}^{*}$ to a map from its compactification $N^{*} \hookrightarrow \mathcal{B}^{*}$ is given by the class $k_{4} \circ j \in H^{4}\left(N^{*}, \mathbf{Z}_{3}\right)=\mathbf{Z}_{3}$, where $f$ is the first map of the Postnikov tower and $j: N^{*} \rightarrow Y_{2}$ is the extension of $f \circ i$. We can compute this by evaluating $k_{4} \circ f$ on $\bar{N}$ : 


$$
\begin{array}{ccccc} 
& & \vdots & & \\
& & Y_{3} & \stackrel{k_{5}}{\longrightarrow} & K(\mathbf{Z}, 5) \\
& \downarrow & & \\
\mathcal{B}^{*}(P) & \stackrel{f}{\longrightarrow} & Y_{2} & \stackrel{k_{4}}{\longrightarrow} & K\left(\mathbf{Z}_{3}, 4\right) \\
\uparrow i & & \uparrow j & & \\
\bar{N} & \hookrightarrow & N^{*} & &
\end{array}
$$

Hence the obstruction is obtained by evaluating $k_{4}$ on $\bar{N}$. This is done by taking codimension two divisors $V_{i}^{\prime} \subset \mathcal{B}^{*}$ representing $\mu^{*}\left(b_{i}\right)$ and computing the intersection numbers with orientations

$$
\frac{1}{4} \sum_{i, j} \bar{q}\left(b_{i}, b_{j}\right) N \cap V_{i}^{\prime} \cap V_{j}^{\prime}=\sum_{i, j} \bar{q}\left(b_{i}, b_{j}\right) N \cap V_{i}^{\prime} \cap V_{j}^{\prime} \quad \bmod 3 .
$$

This gives the other term in our identity.

By the notations suggested in $[R]$

$$
\begin{aligned}
& q\left(\Phi_{d}\right)\left(a_{1}, \ldots, a_{d+2}\right)=\sum_{i<j} q\left(a_{i}, a_{j}\right) \Phi_{d}\left(a_{1}, \ldots, \widehat{a}_{i}, \ldots, \widehat{a}_{j}, \ldots, a_{d+2}\right), \\
& i_{q}\left(\Phi_{d+4}\right)\left(a_{1}, \ldots, a_{d+2}\right)=\sum_{i, j} \bar{q}\left(b_{i}, b_{j}\right) \Phi_{d+4}\left(a_{1}, \ldots, a_{d+2}, b_{i}, b_{j}\right) .
\end{aligned}
$$

we can express the conclusions of Theorem 5.3 as

$$
q\left(\Phi_{d}\right)=i_{q}\left(\Phi_{d+4}\right) \quad(\bmod 3)
$$

i.e. the exterior product of $\Phi_{d}$ with the intersection form is equal to the interior product of $\Phi_{d+4}$ with the intersection form (contraction). We can similarly denote the evaluation of the Pontryagin class as an interior product:

$$
\left(i_{1} \Phi_{d+2}\right)\left(a_{1}, \ldots, a_{d}\right)=\Phi_{d+2}\left(a_{1}, \ldots, a_{d}, 1\right)=2 \Phi_{d}\left(a_{1}, \ldots, a_{d}\right) .
$$

For example if $X$ is simple then $i_{1}^{2}\left(\Phi_{d+4}\right)=4 \Phi_{d}$. The following is a generalization of Theorem 5.2:

Theorem 5.4. If $P \rightarrow X$ is an $S O(3)$-bundle over a closed smooth simply connected 4-manifold, assume that $c_{2}(P)$ is even when $w_{2}(P)=w_{2}(X)$. Then

$$
i_{q}\left(\Phi_{d+4}\right)-(d+1) \Phi_{d+2} \equiv \pm d(d-1) q\left(\Phi_{d}\right)(\bmod 3) .
$$

Proof. $p_{1}-4 \equiv-d-4 \equiv-(d+1) \quad(\bmod 3)$. Hence in case $d \equiv 2(\bmod 3)$ the result follows from Theorem 5.3. Now assume $d \neq 2(\bmod 3)$, then we have $p_{1}\left(P_{k+1}\right)=p_{1}-4 \neq 0(\bmod 3)$. Hence $\pi_{3}\left(\mathcal{B}^{*}\right)_{(3)}=0$ where $\mathcal{B}^{*}=\mathcal{B}^{*}\left(P_{k+1}\right)$. Therefore the ends of $\bar{N}$ are null homotopic, hence we can assume the imbedding $N \subset \mathcal{B}^{*}$ extends to the imbedding of the 4-cycle $N^{*} \subset \mathcal{B}^{*}$. Then Proposition 2.2 implies the result, since the right-hand side of the formula is zero being a multiple of $d(d-1) \equiv 0(\bmod 3)$.

Theorem 5.5. If $P \rightarrow X$ is an $S U(2)$-bundle over a closed smooth simply connected 4-manifold, let $p>3$ be a prime with $c_{2}(P)=(p+1) / 2(\bmod 2)$ when $X$ is even. Then

$$
q^{(p-1) / 2} \Phi_{d} \equiv 0 \quad(\bmod p)
$$

implies

$$
i_{q} i_{1}^{(p-3) / 2}\left(\Phi_{d+2 p-2}\right)+\left[d+\left(3 b_{2}^{+}-1\right) / 2\right] i_{1}^{(p-1) / 2} \Phi_{d+2 p-2} \equiv 0 \quad(\bmod p) .
$$


Proof. Call $c_{2}(P)=k$ and $p=2 m+1$, and pick $a_{1}, \ldots, a_{d+2 m} \in H_{2}(X)$. Consider the bundle $P_{k+m} \rightarrow X$ with Chern class $c_{2}\left(P_{k+m}\right)=k+m$. Hence by hypothesis $c_{2}\left(P_{k+m}\right)$ is odd and we have the $(\bmod p)$ congruences:

$$
p_{1}\left(P_{k+4 m}\right)=-4(k+m)=-d-\frac{3}{2}\left(1+b_{2}^{+}\right)-4 m \equiv-d-\frac{1}{2}\left(3 b_{2}^{+}-1\right)(\bmod p) .
$$

As in the proof of Theorem 5.3 consider the smooth $4 m$ dimensional manifold $N \subset \mathcal{B}^{*}\left(P_{k+m}\right)$ :

$$
N=V_{1} \cap \cdots \cap V_{d+2 m} \cap \mathcal{M}_{k+m}
$$

where $\operatorname{dim}\left(\mathcal{M}_{k+m}\right)=\operatorname{dim}\left(\mathcal{M}_{k}\right)+8 m=2 d+8 m$ and $V_{1}, \ldots, V_{d+2 m}$ are the divisors corresponding to $a_{1}, \ldots, a_{d+2 m}$. Let $N^{*}$ be the Uhlenbeck compactification of $N$, which is a compact stratified space with singular strata

$$
N^{*}-N=\bigcup L_{s}
$$

Each singular stratum $L_{s}$ is a smooth manifold with the $4 s-1$ dimensional link $\Sigma_{s}=S O(3) * S O(3) * \cdots * S O(3)$ consisting of the join of $s$ copies of $S O(3)$ for some $1 \leq s \leq m$. So $\pi_{*}\left(\Sigma_{s}\right)_{(p)}=\pi_{*}\left(S^{4 s-1}\right)_{(p)}$. Since $2 p-3=4 m-1$ by Proposition 4.7 $\pi_{i}\left(\mathcal{B}^{*}\right)_{(p)}=0$ for $4<i<4 m-1$. Hence by obstruction theory we can assume that the imbedding $N \subset \mathcal{B}^{*}\left(P_{k+m}\right)$ extends to the imbedding $N^{*}-L_{m} \subset \mathcal{B}^{*}\left(P_{k+m}\right)$ in the complement of a finite set of points $L_{m}$ (since we are localizing at $p$ we might have to change $N^{*}$ by a relatively prime $p$ multiple of itself). Similar to (7) the number of points of $L_{m}$ is given by

$$
\# L_{m} \equiv\left(q^{m} \Phi_{d}\right)\left(a_{1}, \ldots, a_{d+2}\right) .
$$

So the $\bmod p$ obstruction to extending the imbedding $N^{*}-L_{m} \subset \mathcal{B}^{*}\left(P_{k+m}\right)$ to $N^{*} \subset \mathcal{B}^{*}\left(P_{k+m}\right)$ is

$$
\# L_{m}\left[\Sigma_{s}\right] \in \pi_{4 m-1}\left(\mathcal{B}^{*}\right)_{(p)}=\mathbf{Z}_{p} .
$$

This obstruction can also be computed by evaluating the $k$-invariant on $N$ :

$$
\left\langle k_{2 p-2}, N\right\rangle=\left\langle\Omega \smile \bar{p}_{1}^{m-1}-p_{1}\left(P_{k+4 m}\right) \bar{p}_{1}^{m}, N\right\rangle .
$$

This gives the other term in the statement of the theorem.

Corollary 5.6. If $P \rightarrow X$ is an $S U(2)$-bundle on a closed smooth simply connected simple 4-manifold, let $p>3$ be a prime with $c_{2}(P)=(p+1) / 2(\bmod 2)$ when $X$ is even. Then we have the following implication of $(\bmod p)$ congruences:

$$
q^{(p-1) / 2} \Phi_{d} \equiv 0 \Longrightarrow i_{q}\left(\Phi_{d+p+1}\right)+\left(2 d+3 b_{2}^{+}-1\right) \Phi_{d+p-1} \equiv 0 .
$$

Remark. If we define $\hat{\Phi}_{d}: H_{2}(X) \rightarrow \mathbf{Z}$ by $\hat{\Phi}_{d}(a)=\Phi_{d}(a, \ldots, a)$ we get

$$
\left(q^{m} \hat{\Phi}_{d}\right)(a)=q(a, a)^{m} \frac{(d+2 m) !}{2^{m} d !} \hat{\Phi}_{d}(a)=\frac{q(a, a)^{m}}{2^{m}} \prod_{i=1}^{p-1}(d+i) \hat{\Phi}_{d}(a) .
$$

In particular if $p=2 m+1$ and $d \neq 0(\bmod p)$, then $q^{m} \hat{\Phi}_{d}=0$. So in Theorem 5.5 and Corollary 5.6 if we use the polynomials $\widehat{\Phi}_{d}$ instead of $\Phi_{d}$ we can replace the condition $q^{m} \widehat{\Phi}_{d}=0$ by $d \neq 0(\bmod p)$ in particular:

Corollary 5.7. Let $P \rightarrow X$ be as in Corollary 5.6, then for $d \neq 0(\bmod p)$ :

$$
i_{q}\left(\hat{\Phi}_{d+p+1}\right)+\left(2 d+3 b_{2}^{+}-1\right) \hat{\Phi}_{d+p-1} \equiv 0 \quad(\bmod p) .
$$

If $P \rightarrow X$ is as in Theorem 5.4, then we get a stronger conclusion:

$$
i_{q}\left(\hat{\Phi}_{d+4}\right)+\left(2 d+3 b_{2}^{+}-1\right) \hat{\Phi}_{d+2} \equiv 0 \quad(\bmod 3) .
$$


Remark. We can not determine if $\pi_{1}\left(\mathcal{B}^{*}\right)$ acts trivially on its higher homotopy groups. If this is the case, one can still do the Postnikov decomposition of $\mathcal{B}^{*}$. In particular, in this case we can remove the conditions on $c_{2}(P)$ in all the above results.

\section{SOME APPLICATIONS}

Even though by in large, the Donaldson polynomial invariants of complex surfaces can be computed by other means, the following application demonstrates use of previous theorems, which could apply more general manifolds. Let $d=d_{k}$ (and hence $\left.d_{k+1}=d+4\right)$. As in $[\mathrm{R}]$ by a routine calculation we get

$$
(2 d+n) \Phi_{d}=i_{q} \circ q\left(\Phi_{d}\right)-q \circ i_{q}\left(\Phi_{d}\right)
$$

Invariants $\Phi_{d}\left(a_{1}, \ldots, a_{d}\right)$ are called polynomial invariants since they are elements of the ring of $d$-linear symmetric functions $\operatorname{Sym}^{d}\left(H_{2}(X)\right)$. In terms of a basis $\left\{b_{j}\right\}$ of $\mathrm{H}_{2}(X)$ we can write

$$
\Phi_{d}=\sum \Phi_{d}\left(b_{i_{1}}, \ldots, b_{i_{d}}\right) b_{i_{1}}^{*}, \ldots, b_{i_{d}}^{*} .
$$

For example the intersection form $Q=\sum q\left(b_{i}, b_{j}\right) b_{i}^{*} b_{j}^{*}$ is an element of $\operatorname{Sym}^{2}\left(H_{2}(X)\right)$. Also any element $\beta \in H^{2}(X)$ can be considered to be an element of $\operatorname{Sym}^{1}\left(H_{2}(X)\right)$. By adapting the conventions of [FS] we define the symmetric product $\gamma_{1} \gamma_{2} \in$ $\operatorname{Sym}^{d_{1}+d_{2}}\left(H_{2}(X)\right)$ of two elements $\gamma_{i} \in \operatorname{Sym}^{d_{i}}\left(H_{2}(X)\right), i=1,2$, by

$$
\gamma_{1} \gamma_{2}\left(a_{1}, \ldots a_{d_{1}+d_{2}}\right)=\frac{1}{d_{1} ! d_{2} !} \sum_{\sigma \in S_{d_{1}+d_{2}}} \gamma_{1}\left(a_{\sigma(1)}, \ldots, a_{\sigma\left(d_{1}\right)}\right) \gamma_{2}\left(a_{\sigma\left(d_{1}+1\right)}, \ldots, a_{\sigma\left(d_{1}+d_{2}\right)}\right)
$$

where $S_{d_{1}+d_{2}}$ is the symmetric group on $d_{1}+d_{2}$ letters. For $\gamma \in \operatorname{Sym}^{d}\left(H_{2}(X)\right)$ by convention $\gamma^{(p)} \in \operatorname{Sym}^{2 p}\left(H_{2}(X)\right)$ is defined by

$$
\gamma^{(p)}=\frac{1}{p !} \gamma^{p}
$$

For complex surfaces with "large diffeomorphism group" these polynomials are known to be in the form $[\mathrm{FM}]$ :

$$
\Phi_{d}=\sum_{j=0}^{\left[\frac{d}{2}\right]} m_{d j} Q^{(j)} K^{(d-2 j)}=\sum_{j=0}^{\left[\frac{d}{2}\right]} \tilde{m}_{d j} Q^{j} K^{d-2 j}
$$

where $K=c_{1}(X)$. Here $m_{d j} \in \mathbf{Z}$ and $\tilde{m}_{d j}=m_{d j} / j !(d-2 j)$ !. Since $q\left(Q^{j}\right)=Q Q^{j}=$ $Q^{j+1}$

$$
\begin{aligned}
q\left(\Phi_{d}\right) & =\sum_{j=0}^{\left[\frac{d}{2}\right]} \tilde{m}_{d j} Q^{j+1} K^{d-2 j}, \\
\Phi_{d+4} & =\sum_{j=0}^{\left[\frac{d}{2}\right]+2} \tilde{m}_{d+4, j} Q^{j} K^{d+4-2 j}
\end{aligned}
$$

$$
i_{q}\left(\Phi_{d+4}\right)=\sum_{j=0}^{\left[\frac{d}{2}\right]+2} \tilde{m}_{d+4, j} i_{q}\left(Q^{j} K^{d+4-2 j}\right)
$$


By using (11) we get

$$
\begin{aligned}
i_{q}\left(Q^{j} K^{d+4-2 j}\right) & =i_{q} \circ q\left(Q^{j-1} K^{d+4-2 j}\right) \\
& =q \circ i_{q}\left(Q^{j-1} K^{d+4-2 j}\right)+[2(d+2)+n] Q^{j-1} K^{d+4-2 j} .
\end{aligned}
$$

By iteration we get

$$
\begin{aligned}
i_{q}\left(Q^{\frac{d}{2}+2}\right) & =\left(\frac{d}{2}+2\right)(d+2+n) Q^{\frac{d}{2}+1}, \quad \text { when } d \text { is even, } \\
i_{q}\left(Q^{j} K^{d+4-2 j}\right) & =Q^{j} \tilde{K} K^{d+2-2 j}+2 j\left(d+3-j+\frac{n}{2}\right) Q^{j-1} K^{d+4-2 j} .
\end{aligned}
$$

Here we used $i_{q}\left(K^{r}\right)=\tilde{K} K^{r-2}$ where $\tilde{K}=\sum q^{i j} K\left(b_{i}\right) K\left(b_{j}\right)$, where $q^{i j}=\bar{q}\left(b_{i}, b_{j}\right)$, and $i_{q}(Q)=n$. Also we make the convention that $Q^{-1}=0$ (this term occurs when $j=0$ in the second formula). By substituting these into (13) and equating this new expression of (12) and (13) into the formula of Theorem 5.4 and comparing the corresponding coefficients we obtain the following mod 3 congruence:

$$
\begin{gathered}
2(j+1)(d+2-j+n / 2) \tilde{m}_{d+4, j+1}+\tilde{K} \tilde{m}_{d+4, j}-(d+1) \tilde{m}_{d+2, j} \\
\equiv \pm d(d-1) \tilde{m}_{d, j-1}
\end{gathered}
$$

for $j=0, \ldots,\left[\frac{d}{2}\right]+1$. Here we make the convention $m_{d,-1}=0$. Hence we get

Corollary 6.1. Let $X$ be a simply connected complex surface with a large diffeomorphism group. Let $P \rightarrow X$ be an $S O(3)$-bundle. Assume that $c_{2}(P)$ even when $w_{2}(P)=w_{2}(X)$. Then $\Phi_{d} \bmod 3$ is determined by $\Phi_{d+4}$ or $\Phi_{d+2} \bmod 3$. Conversely, if $\tilde{K} \neq 0$, then $\Phi_{d}$ or $\Phi_{d+2} \bmod 3$ along with the coefficient of the highest term $Q^{\left[\frac{d}{2}\right]+2} K^{d-\left[\frac{d}{2}\right]} \bmod 3$ in $\Phi_{d+4}$ determine $\Phi_{d+4} \bmod 3$.

Since this paper was written Kronheimer and Mrowka have proved a general structure theorem for the Donaldson polynomials of simple manifolds which implies this type of corollary as a special case. Another useful application of these congruences is to show that $\mathbf{C P}^{2}$ is not a simple manifold (a fact which was previously noticed by P. Kronheimer, P. Lisca and D. Koschick). By applying Theorem 5.4 to an $S U(2)$-bundle $P \rightarrow \mathbf{C P}^{2}$ we get that, for $d=0$ or $1 \bmod 3$

$$
\Phi_{d+4}(h, h, h, \ldots, h)=\Phi_{d+4}(1,1, h, \ldots, h) \bmod 3
$$

where $h \in \mathbf{C P}^{2}$ is the generator. Hence if $\mathbf{C P}^{2}$ was simple we would have

$$
\Phi_{d+4}(h, h, h, \ldots, h)=\Phi_{d}(h, \ldots, h) \bmod 3 .
$$

This is a contradiction since for $d=1$ the right-hand side is zero (the space of ASD connections on $\mathbf{C P}^{2}$ of charge $k=1$ is empty [DK]), whereas by Donaldson (for the upper positive cone chamber) the left-hand side is 1 (recall that since $b_{2}^{+}\left(\mathbf{C P}^{2}\right)=1$ in this case, the Donaldson polynomial depends on the chamber structure) $[\mathrm{Mo}]$.

\section{REFERENCES}

[A] S. Akbulut, Torsion in gauge theory, Turkish Math. 19 (1995), 167-177.

[AMR] S. Akbulut, T. Mrowka and Y. Ruan, Torsion classes and a universal constraint on Donaldson invariants for odd manifolds, Trans. Amer. Math. Soc. 347 (1995), 63-76. CMP 95:02

[AS] M. Atiyah and I.M. Singer, The index of elliptic operators IV, Ann. of Math. (2) 93 (1971), 119-138. MR 43:5554 
[D] S. Donaldson, Connections, cohomology and the intersection of four manifolds, J. Differential Geometry 24 (1986), 275-341. MR 88g:57033

[DK] S.K. Donaldson and P. Kronheimer, The geometry of 4-manifolds, Oxford Math. Monographs, Oxford Univ. Press, 1990. MR 92a:57036

[DW] H. Whitney and A. Dold, Classification of oriented sphere bundle over a 4-complex, Ann. of Math. 69 (1959), 667-677. MR 23:A659

[FS] R. Fintushel and R. Stern, 2-torsion instanton invariants, J. Amer. Math. Soc. 6 (1993), 299-339. MR 93f:57038

[FM] R. Friedman and J. Morgan, Smooth four manifolds and complex surfaces, A series of Modern Surveys in Mathematics no. 27, Springer-Verlag, 1995.

[J] I.M. James, The topology of Steifel manifolds, London Math Soc. Lecture Notes, no. 24, Cambridge Univ. Press, 1976. MR 55:4240

[M] G. Masbaum, On the cohomology of the classifying space of the gauge group over some 4-complexes, Bull. Soc. France 119 (1991), 1-31. MR 92a:55009

[Mo] K-C. Mong, Some polynomials on the blow-up of $\mathbf{P}_{2}(\mathbf{C})$, J. Reine Angew. Math. 419 (1991), 67-78. MR 92i:14042

[MT] R.E. Moser and M.C. Tangora, Cohomology operations and applications in homotopy theory, Harper and Row, 1968. MR 37:2223

[T] E. Thomas, Seminar on fiber spaces, Lecture Notes in Math., vol. 13, Springer-Verlag, 1966. MR 34:3582

[To] H. Toda, Composition methods in homotopy groups of spheres, Ann. of Math Stud., no. 49, Princeton Univ. Press, 1962. MR 26:777

Department of Mathematics, Michigan State University, East Lansing, Michigan 48824

E-mail address: akbulut@math.msu.edu 